\title{
Structural involvement in the melting of the charge density wave in $1 \mathrm{~T}-\mathrm{TiSe}_{2}$
}

\author{
Max Burian $\odot,{ }^{1, *}$ Michael Porer, ${ }^{1, *}$ Jose R. L. Mardegan, ${ }^{1, \dagger}$ Vincent Esposito, ${ }^{1, \star}$ Sergii Parchenko,,${ }^{1,}$ Bulat Burganov, ${ }^{2}$

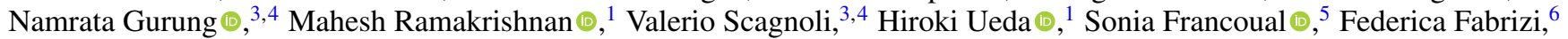 \\ Yoshikazu Tanaka, ${ }^{7}$ Tadashi Togashi, ${ }^{7,8}$ Yuya Kubota, ${ }^{7,8}$ Makina Yabashi, ${ }^{7,8}$ Kai Rossnagel ${ }^{\circ},{ }^{9,10}$ \\ Steven L. Johnson, ${ }^{2,11}$ and Urs Staub ${ }^{1, \|}$ \\ ${ }^{1}$ Swiss Light Source, Paul Scherrer Institute, 5232 Villigen PSI, Switzerland \\ ${ }^{2}$ Institute for Quantum Electronics, ETH Zürich, Auguste-Piccard-Hof 1, 8093 Zürich, Switzerland \\ ${ }^{3}$ Laboratory for Mesoscopic Systems, Department of Materials, ETH Zurich, 8093 Zürich, Switzerland \\ ${ }^{4}$ Laboratory for Multiscale Materials Experiments, Paul Scherrer Institute, 5232 Villigen PSI, Switzerland \\ ${ }^{5}$ Deutsches Elektronen-Synchrotron DESY, Notkestraße 85, 22607 Hamburg, Germany \\ ${ }^{6}$ Diamond Light Source Ltd., Didcot, Oxfordshire OX11 ODE, United Kingdom \\ ${ }^{7}$ RIKEN SPring-8 Center, 1-1-1 Kouto, Sayo-cho, Sayo-gun, Hyogo 679-5148, Japan \\ ${ }^{8}$ Japan Synchrotron Radiation Research Institute (JASRI), 1-1-1 Kouto, Sayo-cho, Sayo-gun, Hyogo 679-5198, Japan \\ ${ }^{9}$ Institut für Experimentelle und Angewandte Physik, Christian-Albrechts-Universität zu Kiel, D-24098 Kiel, Germany \\ ${ }^{10}$ Ruprecht-Haensel-Labor, Deutsches Elektronen-Synchrotron DESY, D-22607 Hamburg, Germany \\ ${ }^{11}$ SwissFEL, Paul Scherrer Institute, 5232 Villigen PSI, Switzerland
}

(Received 25 June 2020; accepted 15 January 2021; published 10 February 2021; corrected 25 February 2021)

\begin{abstract}
In this work, we use ultrafast pump-probe nonresonant and resonant $\mathrm{x}$-ray diffraction to track the periodic lattice distortion and the electronic charge density wave in $1 \mathrm{~T}-\mathrm{TiSe}_{2}$ upon optical excitation. We observe a fluence regime in which the periodic lattice deformation is strongly suppressed but the charge density wave related $\mathrm{Se} 4 p$ orbital order remains mostly intact. Complete melting of both structural and electronic order occurs four to five times faster than expected from a purely electronic charge-screening process, strongly suggesting a structurally assisted weakening of excitonic correlations. Our experimental data provide insight on the intricate coupling between structural and electronic order in stabilizing the periodic-lattice-distortion/charge-density-wave state in $1 \mathrm{~T}-\mathrm{TiSe}_{2}$. The results further show that electron-phonon coupling can lead to different, energy dependent phase-transition pathways in condensed matter systems, opening different possibilities in the conception of nonequilibrium phenomena at the ultrafast scale.
\end{abstract}

DOI: 10.1103/PhysRevResearch.3.013128

\section{INTRODUCTION}

The interplay of electronic and structural order creates a rich playground for the manipulation of properties of condensed matter systems. Indeed, many electronic material properties, such as the macroscopic spin state [1-3] and conductivity [4-6], have a strong, often nonlinear dependence

\footnotetext{
${ }^{*}$ These authors contributed equally to this work.

†Present address: Deutsches Elektronen-Synchrotron DESY, Notkestraße 85, 22607 Hamburg, Germany.

${ }^{*}$ Present address: Stanford Institute for Materials and Energy Sciences, SLAC National Accelerator Laboratory, 2575 Sand Hill Road, Menlo Park, CA 94025.

${ }^{\S}$ Present address: Laboratory for Mesoscopic Systems, Department of Materials, ETH Zurich, 8093 Zürich, Switzerland.

"Author to whom correspondence should be addressed: urs.staub@psi.ch

Published by the American Physical Society under the terms of the Creative Commons Attribution 4.0 International license. Further distribution of this work must maintain attribution to the author(s) and the published article's title, journal citation, and DOI.
}

on the structural order, represented by local displacements of the ions. This dependence is often ascribed to strong electron-phonon coupling [7,8], where tiny changes in atomic positions significantly alter the electronic landscape and vice versa [9-11]. Disentangling the intrinsic electronic from the structurally induced contributions is hence a complex task. It requires specificity to both orders at a time and energy scale where charge- and phonon-driven processes occur: the (ultrafast) femtosecond regime [1-3,5,7-9,12].

A prominent yet controversial system in which the interplay of electronic and structural order is of particular interest is the transition-metal dichalcogenide, titanium diselenide (1T-TiSe ${ }_{2}$ ). Apart from exhibiting doping [13] and pressure [14] induced superconductivity, it shows a commensurate charge-density wave (CDW) at $T<T_{c} \approx 200 \mathrm{~K}$ [15-17]. In its room-temperature (RT) phase, $1 \mathrm{~T}-\mathrm{TiSe}_{2}$ has its electron (Ti) and hole pockets (Se) at the $L$ and $\Gamma$ points of the Brillouin zone, respectively [18-20]. Valence and conduction bands at these Fermi pockets present a small (negative) band gap [19,21], causing charge-carrier accumulation [22] and hence an overall semimetal-like material character $[19,23,24]$ [Figs. 1(a) and 1(b)]. Upon cooling below $T_{c}$, the initial lattice $\left(P_{\overline{3} m 1}\right.$ space group) undergoes a periodic lattice distortion 


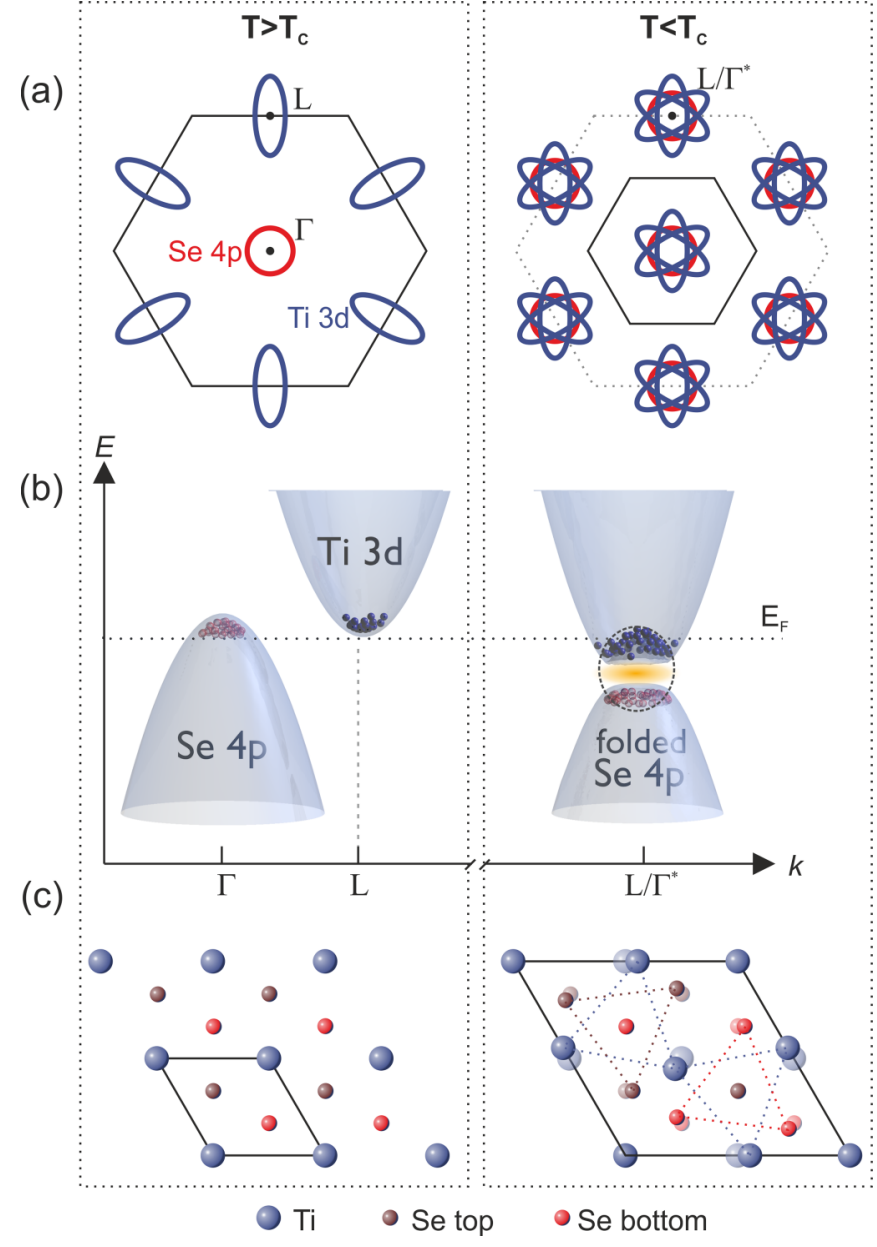

FIG. 1. CDW phase and associated PLD upon cooling of $1 \mathrm{~T}-\mathrm{TiSe}_{2}$. (a) Simplified Fermi surface of a slice through Fermi pockets of holes (red, $\mathrm{Se} 4 p$ ) and electrons (blue, Ti $3 d$ ) in the first Brillouin zone at $\pm A$ along the $k_{c}$ axis, shown in the roomtemperature (left) and CDW (right) phase. The band-folding of Ti $3 d$ and Se $4 p$ states accompanying the CDW transition is indicated, leading to a re-referencing of the Brillouin zone (e.g., the RT $L$ point becomes $\mathrm{CDW} L / \Gamma^{*}$ point). (b) The $3 \mathrm{D}$ representation of the bands illustrates the $p-d$ splitting between the folded Se $4 p$ and Ti $3 d$ bands at $L / \Gamma^{*}$, leading to excitonic correlations between electron-hole pairs (black dotted circle). (c) PLD associated with the CDW phase, causing a doubling of the unit cell along all three dimensions.

(PLD) [25,26] leading to a doubling of the unit cell [Fig. 1(c)] into the $(2 \times 2 \times 2)$ superlattice CDW phase $\left(P_{\overline{3} c 1}\right.$ space group) $[16,25,27]$. This structural doubling folds the Fermi pockets on top of each other, leading to a partial opening of the (p-d) gap at $L / \Gamma^{*}$ [Figs. 1(a) and 1(b)] [28-30] and local distortion of the hybridized orbitals [31]. In short, the phase transition in $1 \mathrm{~T}-\mathrm{TiSe}_{2}$ hence occurs on (i) the electronic, orbital order connected to the CDW and (ii) the structural order relating to the PLD.

While the general picture of the CDW phase transition may be understood qualitatively, the magnitude of electronic and structural contributions remains controversial. One proposed mechanism involves thermally allowed condensation of free carriers (holes and electrons) to bound "excitonic correlated states" that destabilize the room-temperature crystal struc- ture, in turn inducing the PLD [20,27,32,33]. Indeed, charge screening of excitons by photoinjected carriers has been shown to melt the CDW phase and the associated band folding [28,34], indicating the necessity of excitonic correlations in stabilizing the CDW. However, this proposed Bose-Einstein condensation of excitons [35] into a CDW phase with potentially chiral [36], electronic order [37] is highly controversial and is not fully coherent with other experimental observations [38-41]. An alternative origin is suggested by recent calculations, indicating that the PLD is sufficient to energetically stabilize the CDW state [42] and indispensable to reproduce the electronic band structure [31]. Moreover, the observed mixing of phonon and plasmon excitations at $T_{c}$ is a strong evidence for electron-phonon-coupling [9,35], pointing towards a prominent role of structural contributions [43-45].

An ideal technique to investigate systems with coupled electronic and structural order, such as $1 \mathrm{~T}-\mathrm{TiSe}_{2}$, is resonant $\mathrm{x}$-ray diffraction: by tuning the $\mathrm{x}$-ray energy to a core-to-valence electron transition, the polarization dependent scattering cross section is highly sensitive to occupation, geometry, and overall spin of the corresponding valence orbital [46-50]. This becomes especially useful when paired with the ultrashort pulses produced by $\mathrm{x}$-ray free-electron lasers, where an optical laser pump modifies the electronic landscape and a subsequent $\mathrm{x}$-ray pulse probes the change in either orbital [51-53] or structural order [51,54-56], depending on the energy (resonant/nonresonant) or the type of reflection (e.g., charge-symmetry forbidden but orbital allowed). In this work, we use transient resonant/nonresonant $\mathrm{x}$-ray diffraction after near-infrared photoexcitation to disentangle the contributions of structural and orbital order on the melting of the CDW in $1 \mathrm{~T}-\mathrm{TiSe}_{2}$. We find an excitation fluence regime in which the PLD is destabilized while excitonic correlations persist. Further, the suppression of both structural and orbital order occur significantly faster than expected for a pure charge screening induced suppression of excitonic correlations. Our results suggest that at low excitation fluences, the PLD vanishes faster than the buildup of Coulomb screening causing electronic CDW melting, revealing that the relative roles of structural and electronic contributions in ultrafast CDW melting depend on the optical excitation fluence.

\section{EXPERIMENTAL METHODS}

The resonant/nonresonant $\mathrm{x}$-ray diffraction experiment was carried out at the EH2 end station of the BL3 beamline at the SACLA x-ray free electron laser [57]. An illustration of the experimental setup is shown in Fig. 2(a). The sample, an ultrathin $\mathrm{TiSe}_{2}$ single crystal [ $c$ axis out of plane, $61.9 \pm 0.5 \mathrm{~nm}$ thickness-see Figs. 5(a) and 5(c) as well as Appendix A for static sample characterization] in the CDW phase was mounted on a goniometer with a $\mathrm{N}_{2}$ cryostream to stabilize the temperature at approximately $100 \mathrm{~K}$. A slightly focused $(300 \times 300 \mu \mathrm{m}) 35$-fs optical pulse with wavelength $800 \mathrm{~nm}$ excited the sample with a repetition rate of $15 \mathrm{~Hz}$. The horizontal polarized $\mathrm{x}$-ray beam operating at $30 \mathrm{~Hz}$ was focused to a spot size of $10 \times 10 \mu \mathrm{m}^{2}$. During the experiment, the angle between the $\mathrm{x}$-ray- and laser beams was kept fixed. To ensure identical excitation conditions, the diffraction geometries for structural and orbital reflection were kept as 
(a)

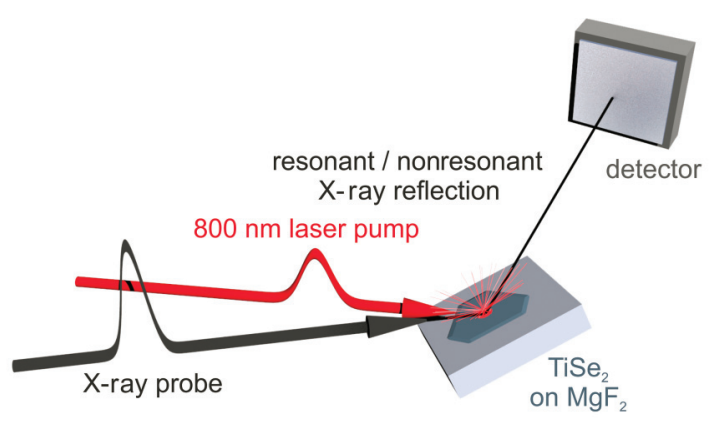

(b)

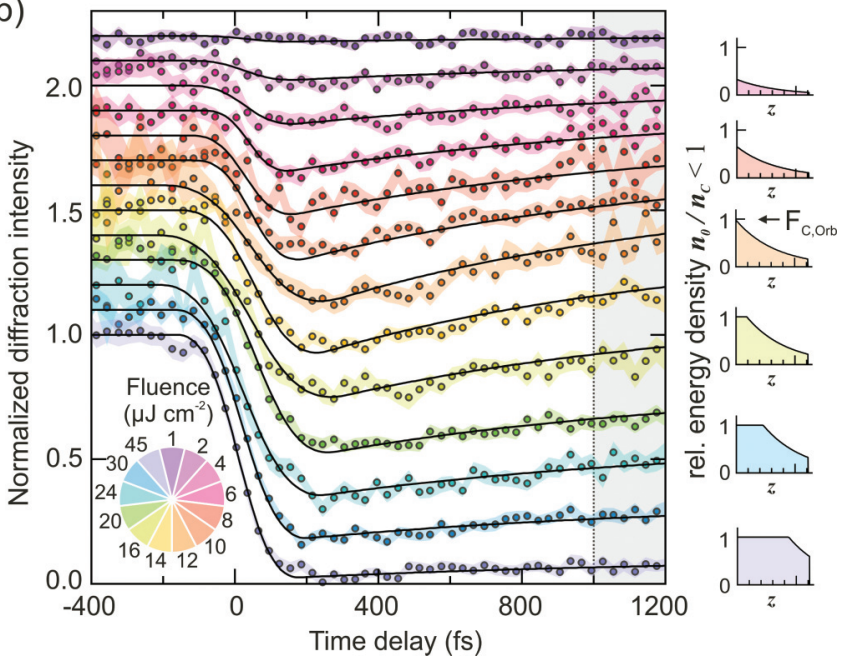

FIG. 2. Experimental overview and XRD transients of the orbital reflection. (a) Schematic representation of the experimental setup.

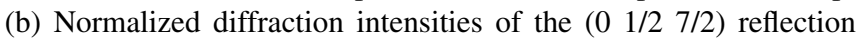
at the Se $K$-edge resonance $(12.652 \mathrm{keV}$ ) (see colored markers for increasing fluence_curves are offset for fluences below $45 \mu \mathrm{J} / \mathrm{cm}^{2}$ for better visibility). Black traces correspond to the orbital order parameter $\eta$ from the global model fit, based on the absorbed energy density profiles at $t=0$ shown on the right. The gray area $(1<t<$ $1.2 \mathrm{ps}$ ) denotes the time region for the analysis in Fig. 4(a).

similar as possible. For the structural reflection, $\mathrm{x}$ rays entered at a grazing angle of $1^{\circ}$ with a sample orientation relative to the horizontal $x$-ray polarization of $\chi=17^{\circ}$, corresponding to a laser incidence angle of $11^{\circ}$. For the orbital reflection, $\mathrm{x}$ rays entered at a grazing angle of $2^{\circ}$ with a sample orientation perpendicular to the horizontal $\mathrm{x}$-ray polarization $\left(\chi=90^{\circ}\right)$, corresponding to a laser incidence angle of $12^{\circ}$. As the sample orientation was different for both reflections, a half-wave plate was used to adjust the polarization of the optical laser to ensure fully $p$-polarized light at the different diffraction geometries. Notably, the effective x-ray penetration depth is significantly larger than the sample thickness of $62 \mathrm{~nm}$, ensuring that the entire sample cross section is probed. As only every second $\mathrm{x}$-ray pulse follows laser excitation, we used shot-to-shot background correction to reduce effects of fluctuations in $\mathrm{x}$-ray pulse intensity. The transients shown in this work come from two-dimensional (2D) integration of the scattering peak on the detector, corrected for the fluorescence background. For orbital and structural reflection we did not observe a shift of the peak maximum but only a decrease in the scattering intensity. The $\mathrm{x}$-ray beam energy was tuned in the vicinity of the Se $K$ edge around $12.65 \mathrm{keV}$ [see Fig. 5(b)

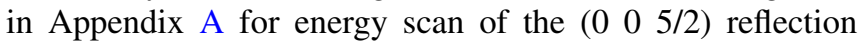
intensity taken at SACLA]. Note that the $x$-ray penetration depth on and off resonance is approximately 15 and $70 \mu \mathrm{m}$, respectively, such that we expect no difference in X-ray attenuation length of the sample thickness of $61.9 \pm 0.5 \mathrm{~nm}$. The inevitable temporal jitter between $\mathrm{x}$-ray and optical laser pulse was measured shot by shot using a transmission-grating based timing tool, which has an accuracy $<10$ fs [full width at half maximum (FWHM)] [58]. The temporal fingerprint of each shot was then used to rebin all data into 30 -fs time segments [59]. In summary, the effective time resolution of the experiment $\Delta t_{\text {Eff }}$ has to take into account (i) the optical laser pulse width $\tau_{L}=35 \mathrm{fs}$, (ii) the x-ray laser pulse width $\tau_{\text {Xray }}=15 \mathrm{fs}$, (iii) the time resolution of the timing tool $\Delta t_{\mathrm{TT}}=10 \mathrm{fs}$, and (iv) the size of data bins $\Delta t_{\text {bin }}=30 \mathrm{fs}$, leading to $\Delta t_{\mathrm{Eff}}=$ $\sqrt{\tau_{L}^{2}+\tau_{\text {xray }}^{2}+\Delta t_{\mathrm{TT}}^{2}+\Delta t_{\text {bin }}^{2}} \approx 50 \mathrm{fs}$.

In the experiment, the optical $(800 \mathrm{~nm})$ laser pulse drives an electronic interband transition in the $\mathrm{TiSe}_{2}$ sample that is in the CDW phase. The excitation injects valence band (Se-VB) electrons from the folded Se $4 p$ orbital as hot carriers into the Ti $3 d$ conduction band (Ti CB). This hot-carrier injection occurs during the pump-pulse duration [28] (here $<35$ fs FWHM). Shifts in plasmon resonance frequency [60], bulk-lattice vibrations $[54,60,61]$, or renormalization of the Ti-CB [34] are observed at slower (50-500 fs) and experimentally observable time scales but depend on the incident laser pump fluence. Indeed, and as we have shown in previous work [60], pump fluences above $0.3 \mathrm{~mJ} / \mathrm{cm}^{2}$ lead to a complete melting of both excitonic and structural order, driven by Coulomb screening-induced exciton breakup. For lower fluences $\left(<0.3 \mathrm{~mJ} / \mathrm{cm}^{2}\right)$, the excited electron plasma is strongly out of equilibrium while a fingerprint of PLD-induced backfolded acoustic-phonon branches appears unaffected [60]. This fluence regime in which electronic and structural order deviate sets the ideal conditions to disentangle the relationship between CDW and PLD.

\section{RESULTS}

We capture the electronic response following excitation by selecting a $(0 \mathrm{k} / 2 \mathrm{l} / 2)$-type reflection at the Se $K$-edge resonance [12.652 keV; Fig. 5(b) of Appendix A]. These reflections are space-group forbidden in the RT and CDW phase for Thomson scattering. They are hence sensitive to the Se $4 p$ orbital order, as shown by (i) the resonance behavior at the Se $K$-edge energy [Fig. 5(b) of Appendix A], (ii) the presence of scattering intensity only in the rotated polarization channel [Fig. 5(c) of Appendix A], and (iii) the absence of scattering above $T_{c}$ [41]. More precisely, the x-ray resonant process at the $K$ edge represents an excitation of a core-state electron to the intermediate $p$-state and back. This process is only nonzero if the wave functions of the two states overlap. Correspondingly, the probed state of interest in this experiment is a projection onto the $1 s$ Se core state, which will be dominated by the Se $4 p$ orbital character, irrespective of its hybridization, as only the $p$-character projection on the Se position will be probed. Further, the phase of the scattering amplitudes from all spherical/symmetric Se $4 p$ orbital 
contributions cancels out, while all noncongruent, aspheric contributions (here at the $P_{\overline{3} c 1}$ Se1 site at Wyckoff position $12 g$ [62]) remain present, giving rise to the diffraction signal (more details can be found in Ref. [41]). As this Se $4 p$ orbital asphericity is caused by site-specific hole condensation [31], this type of resonant reflection directly measures the CDWrelated correlation of bound charges.

Figure 2(b) shows the transient $\mathrm{x}$-ray diffraction (tr-XRD) intensities of the (0 1/2 7/2) orbital reflection. Following the impulsive laser excitation, the diffraction intensity decreases rapidly within $<200 \mathrm{fs}$, reaching almost a complete suppression for an absorbed fluence of $45 \mu \mathrm{J} / \mathrm{cm}^{2}$ [see last trace in Fig. 2(b)] [63]. For fluences below $16 \mu \mathrm{J} / \mathrm{cm}^{2}$, a recovery on the time scale of approximately $>1 \mathrm{ps}$ follows the rapid intensity decrease. For stronger excitations the intensity remains suppressed over the experimental time window-a phenomenon characteristic for phase transitions $[64,65]$. To quantify these experimental observations, we perform a global fit of the time-dependent order-parameter model [52,64], where the order parameter $\eta$ relates to aspheric contributions of the Se $4 p$ orbital geometry (for details see Appendix B). This orbital asphericity parameter $\eta$ reduces to zero if the absorbed local energy density per volume $n(t)$ exceeds a critical threshold $n_{c, \text { Orb }}$, such that the orbital symmetry for $n(t) / n_{c, \text { Orb }}>1$ is the same as found in the RT phase where no excitonic correlations exist. For energy density depth profiles corresponding to selected traces see the right panel of Fig. 2(b).

The experimental data and the model are in excellent agreement [Fig. 2(b)], yielding three significant model parameters. We find an effective optical-absorption length of $34.7 \pm 1.3 \mathrm{~nm}$ compared to the literature value of $17 \mathrm{~nm}$ [66] (see Fig. 7 in Appendix B for a model refined using the literature value). The discrepancy here is likely due to rapid ballistic transport of hot carriers [34] or possibly nonlinear absorption of the 35 fs (FWHM) pump pulse. The model further yields an exponential recovery constant of $\tau_{\text {rel, Orb }}=1.08 \pm 0.03 \mathrm{ps}$, which is in good agreement with electronic thermalization processes [61]. Third, we find complete suppression of the orbital order in the top-most layer for fluences above $12.3 \pm$ $0.5 \mu \mathrm{J} / \mathrm{cm}^{2}\left(\mathbf{F}_{\mathbf{c}, \text { Orb }}\right)$, which corresponds to a local energy threshold to melt the excitonic order of $1.35 \pm 0.05 \mathrm{meV}$ per $\mathrm{Ti}$ atom. Assuming an exponential absorption profile of the laser excitation, this implies that fluences $>33 \mu \mathrm{J} / \mathrm{cm}^{2}$ are required to suppress the orbital order of more than $50 \%$ of the sample volume. This compares well with the previously (not depth resolved) determined threshold of approximately $40 \mu \mathrm{J} / \mathrm{cm}^{2}$ for switching to normal-state conductivity caused by breakup of excitonic correlations [60,61]. This indicates that the observed melting of the Se $4 p$ orbital order is linked to the reduction of the correlated nature of bound holes as breakup of excitonic correlations suppresses hole-induced orbital distortion and hence reduces the aspheric scattering contribution [67].

In order to directly compare melting of the orbital order to dynamics of the PLD, we further acquired nonresonant $(12.620 \mathrm{keV})$ transient tr-XRD traces for a CDW space-group allowed $[(h / 2 k / 2 l / 2)$ type] reflection. This reflection is sensitive to contributions of both $A 1 g^{*}$ and $E g^{*}$ modes that soften at the $L$ point during the RT- to CDW-phase transition

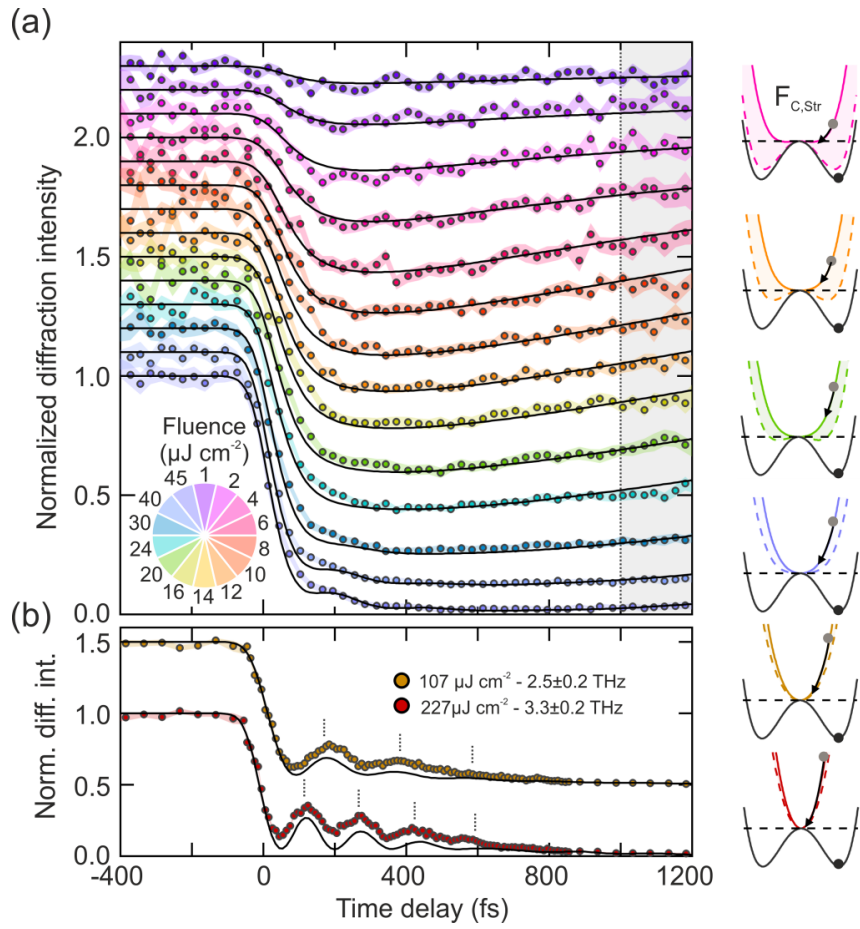

FIG. 3. XRD transients of the structural reflection. (a) Normalized diffraction intensities of the (5/2 1/2 3/2) reflection (see colored markers) below the Se $K$ edge $(12.620 \mathrm{keV})$ with increasing fluence (curves are offset for fluences below $45 \mu \mathrm{J} / \mathrm{cm}^{2}$ for better visibility). The gray area denotes the time region for the analysis in Fig. 4(a). (b) Diffraction intensities after high-fluence excitation, showing a strong oscillatory component (dashed markers note half period). For (a) and (b), black traces correspond to transients calculated from the double-well-potential model. On the right, energy potentials for selected traces at $t=0$ are shown (black: ground state; solid color: excited potential in top layer; dashed color: excited potential at backside of sample).

[68-70], such that the diffraction intensity is zero if the PLD is suppressed.

Figure 3(a) shows the tr-XRD intensities of the (5/2 1/2 $3 / 2$ ) structural peak after excitation. Similar to the orbital reflection shown in Fig. 2(b), the scattering signal decreases rapidly within $<200 \mathrm{fs}$, reaching near complete suppression for fluences at $40 \mu \mathrm{J} / \mathrm{cm}^{2}$ [bottom trace in Fig. 3(a)]. Also here, the scattering intensity partially recovers for fluences below $10-16 \mu \mathrm{J} / \mathrm{cm}^{2}$, while a slight oscillatory component appears in the $0-400$-fs time window for fluences $>25 \mu \mathrm{J} / \mathrm{cm}^{2}$. With a further increase in excitation energy above $100 \mu \mathrm{J} / \mathrm{cm}^{2}$ [Fig. 3(b)] the oscillatory contribution both increases and becomes more coherent, while shifting in frequency from $2.5 \pm 0.2$ to $3.3 \pm 0.2 \mathrm{THz}$ for 107 and $227 \mu \mathrm{J} / \mathrm{cm}^{2}$, respectively. On a first glance, these frequencies appear to be in good agreement with the $E g^{*}(2.2 \mathrm{THz})$ and $A 1$ $g^{*}(3.4 \mathrm{THz})$ phonon modes $[54,61,68]$, which are the in-phase and out-of-phase amplitude modes of the CDW phase [71]. This modulation is well represented by a displacive "phonon" excitation. Yet, both amplitude modes show a significant mode-softening throughout the CDW- to RT-phase transition [69] such that the observed hardening with increasing fluence appears counterintuitive. 
(a)

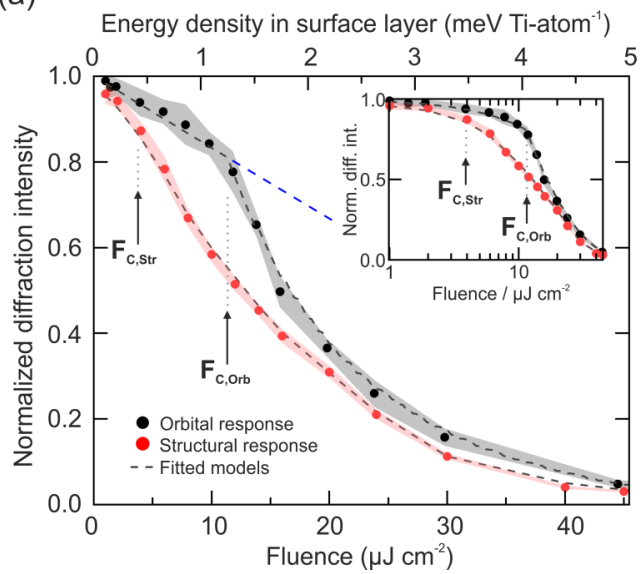

(b)

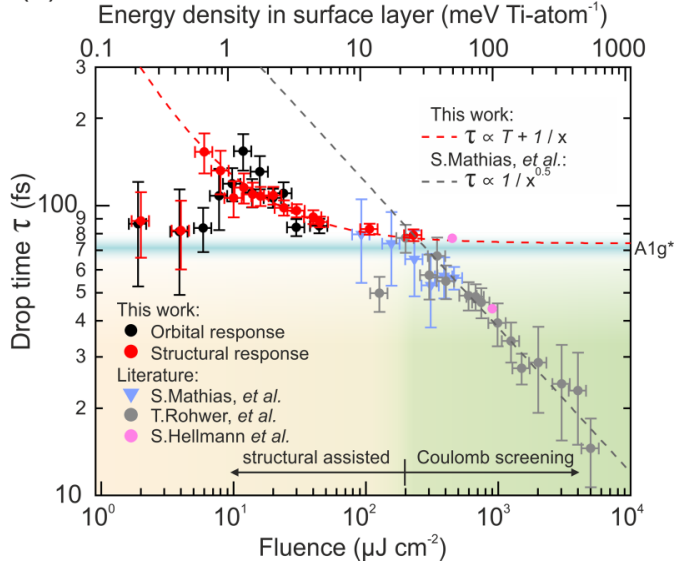

FIG. 4. Fluence dependence of orbital and structural order. (a) Averaged tr-XRD intensity of orbital (black) and structural (red) order in the 1.0-1.2 ps time window [gray regions in Figs. 2(b) and 3(a)]. The blue dashed line indicates deviation of the orbital response from the linear fluence dependency, marked by orbital threshold energy $\mathbf{F}_{\mathbf{c}, \text { Orb }}$. The inset shows a logarithmic representation to highlight the transition in structural response at $\mathbf{F}_{\mathbf{c} \text {,Str }}$. (b) Comparison of the dynamics of orbital and structural response with literature (Mathias et al. [34], Rohwer et al. [28], and Hellmann et al. [72]). In the high-fluence regime ( $\left.>200 \mu \mathrm{J} / \mathrm{cm}^{2}\right)$, hot-carrier screening of excitonic correlations is sufficiently fast to dominate the overall melting dynamics. In the low-fluence regime $\left(<200 \mu \mathrm{J} / \mathrm{cm}^{2}\right)$, structurally assisted destabilization following the soft mode outpaces charge screening and hence becomes the governing mechanism in melting of the CDW phase.

To describe the structural dynamics in the low $\left(<45 \mu \mathrm{J} / \mathrm{cm}^{2}\right)$ and high $\left(>100 \mu \mathrm{J} / \mathrm{cm}^{2}\right)$ fluence regime, we formulate a model based on a double-well potential (DWP) [51,73] describing the $A 1 g^{*}$ potential energy landscape, as depicted by the black trace in the right panel of Fig. 3. Prior to excitation, the system resides at the minimum positions corresponding to the equilibrium PLD in the CDW phase. Hot-carrier injection through laser excitation causes an instantaneous, transient change in the ionic potential energy landscape [9], resulting in coherent atomic oscillations around the new, shifted potential minimum (see Appendix $\mathrm{C}$ for a detailed description). Following the initial excitation, the potential energy landscape relaxes slowly back to its original shape with an exponential time constant $\tau_{\text {rel,Str }}$.

Again, the model agrees well with the experimentally obtained tr-XRD data (Fig. 3). To be consistent with the fit to the orbital reflection transients, we fix the effective opticalabsorption length to $34 \mathrm{~nm}$ [74]. The exponential recovery parameter $\tau_{\text {rel,Str }}$ is $1.6 \pm 0.2 \mathrm{ps}$, which is slightly longer than the $1.08 \pm 0.03$ ps obtained from the analysis of the orbital reflection transients and compares well to the relaxation of coherent structural dynamics in similar systems [51]. Further, we find suppression of the ground-state potential barrier for local energy densities $>0.52 \pm 0.06 \mathrm{meV}$ per $\mathrm{Ti}$ atom, which is reached in the top-most layer for fluences $>4.8 \pm 0.5 \mu \mathrm{J} / \mathrm{cm}^{2}$ $\left(\mathbf{F}_{\mathbf{c} \text {,Str }}\right)$ (see pink schematic in the right panel of Fig. 3) [i.e., when the transient equilibrium coordinate of the $\left(P_{\overline{3} c 1}\right) \mathrm{CDW}$ amplitude mode becomes zero]. Further increase in fluence leads to a larger sample fraction with suppressed energy barrier and a further steepening of the potential-barrier wall for strongly excited regions near the surface. The lattice, following the landscape given by the single-well potential, now traverses through the high-temperature structure and overshoots, yielding an oscillating, CDW-like distorted lattice with an inverting amplitude at each zero crossing. We observe the oscillation at twice its frequency as the diffraction intensity scales with the squared amplitude. This is a coherent oscillation of a CDW-related $L_{1}$ soft-phonon [68] of the RT $\left(P_{\overline{3} m 1}\right)$ structure [Fig. 3(b)]. As the potential walls become steeper with increasing fluence, this $L_{1}$-mode-related oscillation effectively hardens - a transient analog to the thermal behavior when temperature increases above $T_{\mathrm{c}}[68,69]$.

\section{DISCUSSION}

The comparison of results obtained from the on- and offresonance transient data suggests two distinct pathways for melting of structural and orbital order, as evidenced by the different critical thresholds of 0.52 and $1.35 \mathrm{meV} / \mathrm{Ti}$ atom, respectively. This difference is found not only in the quantitative model parameters, but also becomes directly apparent in the experimental data, particularly in the 1-1.2-ps time window [gray regions in Figs. 2(b) and 3(a)]. In this time regime (i) oscillatory contributions in the structural response have vanished and (ii) the orbital response is mainly sensitive to the sample fraction with energy densities above $\mathbf{F}_{\mathbf{c}, \text { Orb }}$, as contributions from lower densities have decayed by $1 / e$. As is apparent in Fig. 4(a), the scattering intensity of the structural reflection (red dots) decreases continuously with the pump fluence up to approximately $20 \mu \mathrm{J} / \mathrm{cm}^{2}$ [see transition region in inset of Fig. 4(a)]. In contrast, fluence dependence of the intensity of the orbital reflection (black dots) shows a nonlinear behavior [see blue dashed line in Fig. 4(a) for deviation from linear], with the pivot point coinciding with the orbital threshold $\mathbf{F}_{\mathbf{c} \text {,Orb }}$. Remarkably, at this fluence the structural signal has already decreased by approximately $50 \%$, in agreement with its significantly lower structural threshold $\mathbf{F}_{\mathbf{c} \text {,Str }}$. It is hence evident that at energy densities below 1.35 $\mathrm{meV} / \mathrm{Ti}$ atom, the PLD is significantly destabilized while charge carrier correlations remain mostly intact.

These results point toward a structurally assisted melting of carrier correlations. Hot-carrier and hole injection distorts the 
local energetic landscape [9], causing a structural rearrangement along the $A 1 g^{*}$ trajectory towards the RT phase. Note that this structural rearrangement also occurs for fluences below the orbital threshold $\mathbf{F}_{\mathbf{c} \text {, Orb }}$, in a state where carrier correlations are not yet molten by the pump. As the CDW phase is not stable in the absence of a PLD, the structural movement towards the RT phase lowers the activation barrier for exciton breakup towards the observed threshold through altering the electronic band structure [31]. Surpassing this activation barrier, the melting of carrier correlations initiates a self-amplifying cascade of band-edge renormalization and self-injection through hot-carrier relaxation [34], as evidenced by the nonlinear fluence dependence of the orbital order [Fig. 4(a)].

Indeed, this scenario, in which the absence of the PLD destabilizes excitonic correlations, is plausible only if the transient structural motion is sufficiently fast compared to the competing channel of Coulomb screening [9,28,34]. A comparison of the exponential diffraction intensity drop time $\tau$ of our data with the time constants for the transients of the folded Se $4 p$ occupation determined by angle-resolved photoemission spectroscopy (ARPES) [28,34] is shown in Fig. 4(b) (for corresponding fits with the double-exponential model taken from literature [28,34] see Fig. 6(a) of Appendix B and Fig. 10 of Appendix C). Obviously, the orbital and structural responses in the low-fluence regime are significantly faster than the CDW melting processes due to Coulomb screening induced exciton breakup [see that the gray dotted line in Fig. 4(b) for the Fröhlich-law prediction [28,34] lies above our experimental data]. All transients we observe are slower than $75 \mathrm{fs}$, which corresponds to a quarter oscillation of the $A 1 g^{*}$ soft mode (that is argued to be the bottleneck to switch the lattice from the CDW to the RT phase [61]). These two findings strongly suggest that structural destabilization of the PLD is the dominant, rate-limiting process in the low-fluence regime. For fluences above approximately 20 $\mathrm{meV} / \mathrm{Ti}$ atom, charge screening is sufficiently fast to outpace the phononic response [28,34], such that the corresponding $\mathrm{THz}$ fingerprint remains present despite the absence of excitonic correlations [60]. Indeed, for the highest fluences tested, the coherent oscillations of the structural order with the CDWassociated soft mode implies that the periodicity associated with the PLD remains existing in an oscillatory state before the mode is damped out and the corresponding scattering intensity finally approaches zero. This may explain the observation of persistent back-folded acoustic-phonon branches in the high excitation regime [60] despite the presence of a structural single-well potential. For fluences below approximately $20 \mathrm{meV} / \mathrm{Ti}$ atom, the structural response outpaces the buildup of charge screening, such that removal of the PLD destabilizes excitonic correlations $[43,44]$ and leads to structurally assisted melting of the CDW phase. Finally, and as further affirmation, the here observed melting thresholds of 0.52 and $1.35 \mathrm{meV} / \mathrm{Ti}$ atom are close to the predicted energy differences between the structural CDW and RT phases of a few meV/Ti atom [75,76] (particularly when taking the sample temperature of $100 \mathrm{~K}$ into account).

On a final note, we point out two crucial aspects to frame the context of our findings. For one, the structural and orbital melting thresholds of 0.52 and $1.35 \mathrm{meV} / \mathrm{Ti}$ atom correspond to excitation densities of $1.3 \times 10^{19}$ and $0.5 \times$ $10^{19}$ photons $/ \mathrm{cm}^{3}$ (for comparison: the density of electrons and holes in the CDW pair state is $4.2 \times 10^{19} \mathrm{~cm}^{-3}$ [77]). This means that at the structural and orbital melting thresholds, a single photon is absorbed in a volume of approximately 350-150 CDW-phase unit cells. For the other, tr-XRD as well as tr-ARPES (as, e.g., used in Refs. [28,34,72]) both measure only long-range ordered phenomena-e.g., a change in the electronic potential or structure of a single unit cell within the absorption volume of 350-150 CDW-phase unit cells would not be detectable. Both of these aspects show that our observations must be seen in a macroscopic context and are hence not directly comparable to microscopic predictions. While, e.g., it is has recently been predicted that electronic excitation may destabilize CDW and PLD on the microscopic single unit cell scale [9], we find that the macroscopic phase transition of the bulk sample can either be structurally (low fluence) or electronically (high fluence) dominated. Indeed, this is directly in line with the seminal work by Rohwer et al. [28] on charge-screening induced CDW melting, as buildup of the electron-hole plasma is a macroscopic multibody effect [78].

\section{CONCLUSION}

Our work provides experimental evidence that the PLD is a crucial ingredient in (de)stabilizing the CDW phase in $1 \mathrm{~T}-\mathrm{TiSe}_{2}$. It is further evident that the kinetics of competing processes seem to determine whether Coulomb screening from injected hot carriers or structural destabilization of the PLD triggers the overall melting of the macroscopic CDW phase. Yet regardless of this initial trigger, our results show that both electronic and structural order melt quasisimultaneously, confirming the presence of strong electron-phonon coupling in the 1T-TiSe ${ }_{2}$ system [9]. Interestingly, the laser excitation appears not to be sufficiently strong to directly drive a macroscopic electronic transition (i.e., Coulomb screening). Yet it may still distort the local electronic potential that stabilizes the PLD, thereby initiating an expanding cascade of electron-phonon-coupling mediated transitions as a macroscopic effect. We anticipate similar, potentially even more drastic, effects within the wide range of compounds that present strong coupling between different orders, such as, e.g., cuprates $[4,10,79]$, other dichalcogenides $[6,72,80]$, (complex) oxides $[1,3,81]$, as well as superlattice $[82,83]$ and spin-ice $[84,85]$ systems.

For the specific system of $1 \mathrm{~T}-\mathrm{TiSe}_{2}$, our findings open a range of new questions that require further experimental efforts. For one, a detailed fluence dependence over the structurally assisted and the Coulomb screening energy regime would rule out experimental deviations that one may encounter when comparing literature data. Here, particular importance has to be paid to the initial temperature before the excitation, as a change in thermal condition may add an energetic offset and directly affect the correlated carrier concentration [77], which will both likely affect the optical melting threshold. For the other, we note that the structural reflection used in this work is mainly sensitive to in-plane contributions of the $A 1 g^{*}$ mode. Including a reflection with sensitivity to out-of-plane displacements [58] might allow us 
(a)

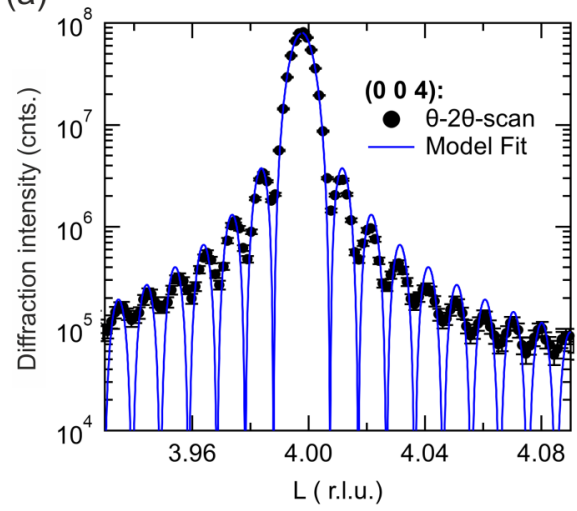

(b)

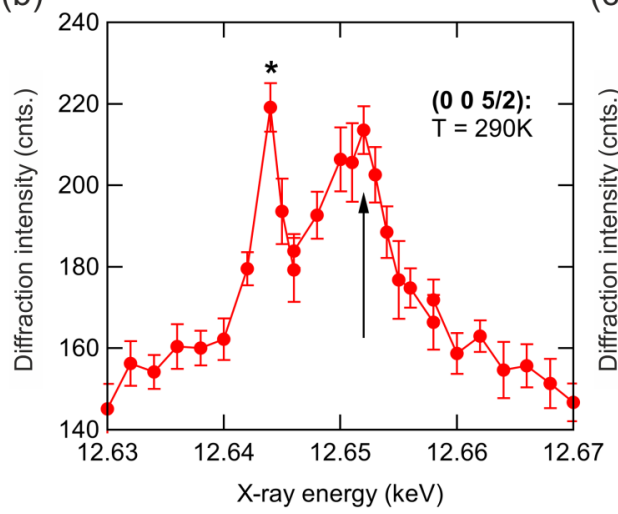

(c)

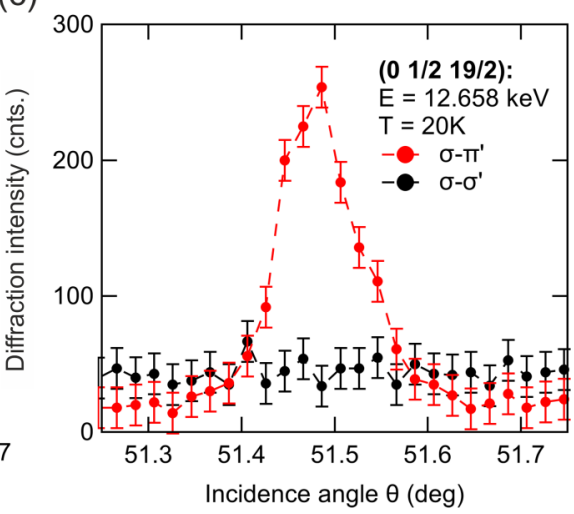

FIG. 5. Static characterization of the TiSe 2 nanoflake. (a) X-ray diffraction intensity around the specular (004) (RT phase) reflection. Clear Laue oscillations from the finite sample thickness can be observed. Fitting of the rocking curve yields a film thickness of $61.9 \pm 0.5 \mathrm{~nm}$.

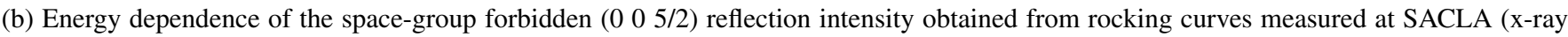
fluorescence background was subtracted from scattering images before data-integration). The black arrow indicates the x-ray energy at which resonant scattering experiments where performed $(12.652 \mathrm{keV})$. Nonresonant x-ray-scattering experiments were performed $\sim 30 \mathrm{eV}$ pre-edge, so at $12.620 \mathrm{keV}$. The sharp peak at $\sim 12.645 \mathrm{keV}$ (see* mark) is likely a multiple-scattering artefact. (c) Static resonant x-ray-diffraction scan of the incidence angle $\theta$ through the (0 1/2 19/2) reflection at an energy of $12.658 \mathrm{keV}$, using a high-resolution Si (111) analyzer. No Thomson scattering is observed in the $\sigma-\sigma^{\prime}$ channel. In contrast, strong scattering from the Se $4 p$ orbital asymmetry (also called ATS scattering) is recorded in the $\sigma-\pi^{\prime}$ scattering channel. For a detailed characterization of the resonant scattering behavior of the corresponding peak family, see Ueda et al. [41].

to reconstruct the precise PLD trajectory upon destabilization, which would give further insight on electron-phonon-coupling anisotropy [9] and the related phonon-mode overdamping [61].

Experimental and model data are accessible from the Materials Cloud Archive [86].

\section{ACKNOWLEDGMENTS}

Static X-ray characterization of the sample was performed at the P09 beamline (PETRA III) and X04SA beamline Swiss Light Source (SLS) during in-house access as well as the I16 beamline (Diamond Light Source) under Proposal No. MT15742. The time-resolved x-ray experiments were performed under the approval of the Japan Synchrotron Radiation Research Institute (JASRI Proposal No. 2017B8039). We thank C. Monney, T. Jaouen, and P. Beaud for enlightening discussions. The research leading to these results has received funding from the Swiss National Science Foundation and its National Centers of Competence in Research, NCCR MUST No. 51NF40-183615 and NCCR MARVEL. This research was supported by the Swiss National Science Foundation (Grant No. 200021_169698). The research leading to this result has also been supported by the project CALIPSOplus under Grant Agreement No. 730872 from the EU Framework Programme for Research and Innovation HORIZON 2020.

\section{APPENDIX A: SAMPLE CHARACTERIZATION}

Multiple $\mathrm{TiSe}_{2}$ nanoflake samples were produced by repetitive exfoliation from a bulk single crystal grown by iodine vapor transport. The final sample used for time resolved experiments [size: $400 \times 500 \mu \mathrm{m}$; thickness $=62 \mathrm{~nm}$; see Fig. 5(a)] was van der Waals bonded to an $\mathrm{MgF}_{2}$ substrate that possesses comparable thermal expansion coefficients and minimizes thermally induced strain on the flake. Static x-ray diffraction to determine the $\mathrm{TiSe}_{2}$ nanoflake thickness was carried out at the X04SA (MS) beamline of the Swiss Light Source (SLS); see Fig. 5(a). Static resonant x-ray diffraction to screen and select appropriate reflections for transient measurements was performed at the I16 beamline of the Diamond Light Source as well as at the P09 beamline at PETRAIII at DESY using samples of up to $5 \mu \mathrm{m}$ thickness; see Fig. 5(c).

\section{APPENDIX B: FITTING OF ORBITAL ORDER TRANSIENTS}

We describe the dynamics of the orbital order using a simplified single time-dependent order-parameter model, which has initially been developed to quantitatively describe arbitrary phase transitions upon excitation [64]. For a detailed model description, we refer the reader to Beaud et al. [64] the following gives a brief outline necessary to understand the parameters derived in this work. A summary of all model parameters can be found in Table I.

Here, the asphericity parameter $\eta$ is a direct measure of the Se $4 p$ orbital order in the CDW phase. In the unexcited CDW phase, where the presence of excitonic correlations induces a Se $4 p$ orbital distortion [31], the order parameter is normalized to $\eta=1$. Upon (i) heating the sample above $T_{c}$ (at approximately $200 \mathrm{~K}$ ) or (ii) sufficiently strong optical excitation, excitonic correlations, and hence the Se $4 p$ orbital distortion vanish, such that the order parameter $\eta$ reduces to zero. In quantitative terms, we correlate the order parameter $\eta$ with the laser-induced energy per volume $n$ : if the deposited energy is above a critical threshold energy $n_{c, \text { Orb }}$, the phase transition from the orbital-ordered $(\eta=1)$ to the nonorbital-ordered $(\eta=0)$ phase occurs. Following the literature $[52,64,65]$, we formulate this phenomenological correlation 
TABLE I. Overview of all model parameters used to fit the orbital order transient dynamics.

\begin{tabular}{clll}
\hline \hline Variable & Unit & \multicolumn{1}{c}{ Description } & Equation \\
\hline$n_{c, \text { Orb }}$ & meV/Ti atom & critical threshold energy density of the orbital order & (B2) \\
$n$ & meV/Ti atom & laser-induced energy density & (B3) \\
$\varepsilon$ & & scaling exponent: scattering-intensity and order parameter & (B2) \\
$\tau_{\text {rel, Orb }}$ & relaxation-time constant of the orbital order & (B3) \\
$z_{a}$ & $\mathrm{Nm}$ & optical-absorption length of the 800-nm pump & (B4) \\
$\tau_{L}$ & $\mathrm{Fs}$ & pulse length of the optical laser (35 fs) & (B4) \\
$F$ & $\mathrm{~J} / \mathrm{cm}^{2}$ & absorbed laser fluence & \\
\hline \hline
\end{tabular}

according to

$$
\eta=\left(1-\frac{\min \left(n, n_{c, \text { Orb }}\right)}{n_{c, \text { Orb }}}\right)^{\varepsilon},
$$

where the exponent $\varepsilon$ determines the scaling relation between the scattering intensity $I$ and the order parameter (note that $I \propto \eta^{2}$, so $\varepsilon=0.5$ for a linear dependency). To account for (a) the optical excitation profile and (b) partial recovery of the orbital order within the experimental time window [Fig. 2(b)], we generalize the relation in Eq. (B1) to account for (a) spatial inhomogeneity and (b) time-dependent energy dissipation according to

$$
\eta(t, z)=\left(1-\frac{\min \left(n(t, z), n_{c, \mathrm{Orb}}\right)}{n_{c, \mathrm{Orb}}}\right)^{\varepsilon} .
$$

Here, $n(t, z)$ includes the relaxation constant $\tau_{\text {rel, Orb }}$ as well as the time- and space-dependent initial excitation profile $n_{0}(t, z)$, yielding

$$
n(t, z)=n_{0}(t, z) e^{-\frac{t}{\tau_{\mathrm{rel}, \mathrm{Orb}}}} .
$$

As the optical-absorption length $z_{a}$ of the 800-nm pump is smaller than the sample thickness, we must correct for inhomogeneous laser absorption of the initial excitation $n_{0}(t, z)$. We hence define the excitation profile along $z$ and divide the 60-nm-thick flake into $N$ slices of thickness $d z$ (here, $d z=0.6 \mathrm{~nm}$ ) such that the initial $z$-dependent absorbed energy density is

$$
\begin{aligned}
n_{0}\left(t, z_{i}\right) d z= & F\left(1-e^{-d z / z_{a}}\right) e^{-z_{i} / z_{a}} \\
& \times \frac{1}{2}\left[1+\operatorname{erf}\left(\frac{t \sqrt{4 \ln 2}}{\tau_{L}+\tau_{m, \text { Orb }}}\right)\right],
\end{aligned}
$$

where $F$ is the absorbed laser fluence impeding on the sample, corrected for geometry and polarization dependent reflection losses. Note that $F$ gives the area density of the laser in units of $\mathrm{J} / \mathrm{cm}^{2}$, whereas $n_{0}\left(t, z_{i}\right)$ gives the energy density per volume in units of $\mathrm{J} / \mathrm{cm}^{3}$. Taking into account the unit cell dimensions of $\mathrm{TiSe}_{2}$ and the laser wavelength $(800 \mathrm{~nm}$ corresponding to $1.55 \mathrm{eV}$ ), the obtained energy density profile $n_{0}\left(t, z_{i}\right)$ is converted into atomic units of meV/Ti atom. The critical fluence $F_{c}$, Orb $\left(\mathrm{J} / \mathrm{cm}^{2}\right)$ hence relates to the scenario where the critical energy density $n_{c, \text { Orb }}(\mathrm{meV} / \mathrm{Ti}$ atom) is reached in the top surface layer of the sample.

Equation (B4) further contains a second, time-dependent term which accounts for the finite pulse length of the optical pump laser of $\tau_{L}=35 \mathrm{fs}$ (FWHM) as well as the orbital order melting dynamics $\tau_{m \text {, Orb }}$. Both contributions are assumed to have a Gaussian temporal profile following the error function erf. The time-dependent change in scattering intensity is then given by

$$
\frac{I(t)}{I_{0}}=\left|\frac{1}{N} \sum_{i=0}^{N}\left(1-\frac{\min \left(\left(n_{0}\left(t, z_{i}\right) e^{\left.\left.-t / \tau_{\mathrm{rel}, \mathrm{Orb}}\right), n_{c, \mathrm{Orb}}\right)}\right.\right.}{n_{c, \mathrm{Orb}}}\right)^{\varepsilon}\right|^{2} .
$$

Finally, we correct for the time resolution given by the length of the x-ray probe pulse (15 fs, FWHM) by convolution of each time trace $\frac{I(t)}{I_{0}}$ with the corresponding Gaussian function.

During the global fitting procedure, we calculate the change in scattering intensity for all experimental fluences according to Eq. (B5) and simultaneously compare them to all experimental traces, resulting in $\varepsilon=0.57 \pm 0.03), \tau_{\text {rel, Orb }}=$ $1.08 \pm 0.03 \mathrm{ps}, n_{c, \text { Orb }}=1.35 \pm 0.05 \mathrm{meV}$ per Ti atom and $z_{a}=34.7 \pm 1.3 \mathrm{~nm}$ with a reduced $\chi^{2}=1.46$. All traces together with the superimposed fit can be seen in Fig. 2(b). Here, the obtained value $\varepsilon=0.57 \pm 0.03$ relates to an almost linear $(\varepsilon=0.5)$ relation between the orbital order and the observed scattering intensity.

The results of the orbital order melting dynamics $\tau_{m \text {,Orb }}$ for each diffraction transient are shown in Fig. 6(b). Note that the values extracted for $\tau_{m \text {,Orb }}$ correspond to the FWHM of the signal drop and are hence not directly comparable to other literature results [28,34] (which are based on a doubleexponential model fit where $\tau_{m \text {,orb }}$ denotes a $1 / e$ intensity decrease/increase). In order to compare our data to literature, we refitted all data with such a double exponential fit, as shown in Fig. 6(a): a correlation analysis of the FWHM and 1/e signal drop times shown in Fig. 6(c) shows a clear linear relation scaling by $0.66 \pm 0.10$, closely matching the ideal FWHM to $1 / e$ conversion factor of 0.67 .

Notably, the more complex time dependent orderparameter model from Ref. [56] includes an additional term to describe the time-independent sample response to excitation at infinite time and assuming no heat dissipation $[64,65]$. Here, this time-independent contribution was always found to be less than $1 \%$, which is why we excluded this contribution from the model as presented above.

It should further be noted that the fitted optical-absorption length $z_{a}$ of $34.7 \pm 1.3 \mathrm{~nm}$ is approximately twice as large as suggested in literature (17 nm) [66]. We therefore initially tried to model the experimental data by keeping this parameter fixed to the reported value during the refinement process. While we were able to describe single traces at a time, a global fit of all traces simultaneously never converged to agreement 

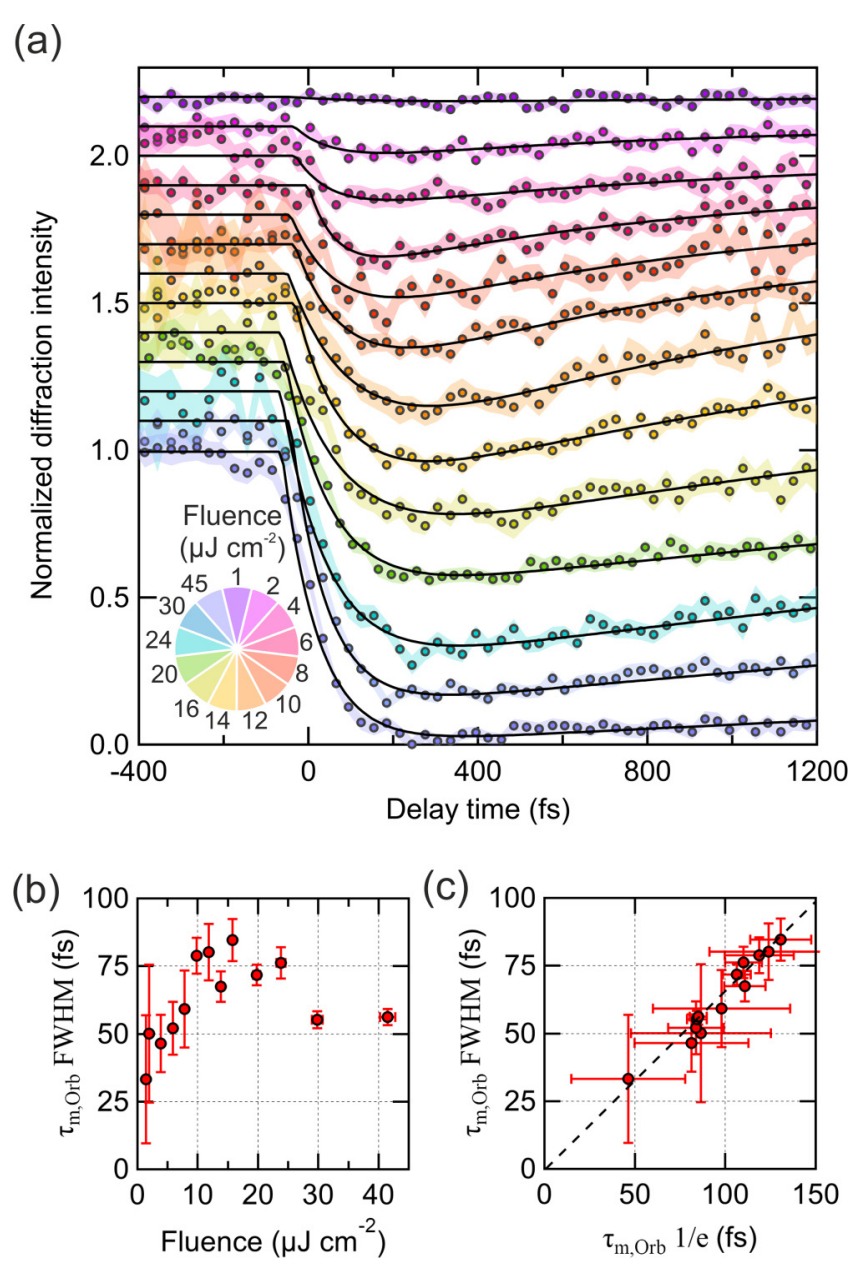

FIG. 6. Double-exponential model fit of the orbital order XRD transients. (a) Normalized XRD transients of the orbital (0 1/2 $7 / 2$ ) reflection at $12.652 \mathrm{keV}$ (see colored markers for increasing fluence-curves are offset for fluences below $45 \mu \mathrm{J} / \mathrm{cm}^{2}$ for better visibility) together with the fits (black curves) of the doubleexponential model according to literature [28,34]. (b) The parameter of the orbital order melting dynamics $\tau_{m, \text { Orb }}$ as extracted from the single order-parameter model as shown in Fig. 2(b) for different fluences. (c) Correlation analysis of the $\tau_{m \text {,Orb }}$ extracted from the single order-parameter model (vertical axis) and the double-exponential model (horizontal axis). The dashed black line is a linear fit to the data points - the slope corresponds to $0.66 \pm 0.10$, closely matching the ideal FWHM to $1 / e$ conversion factor of 0.67 .

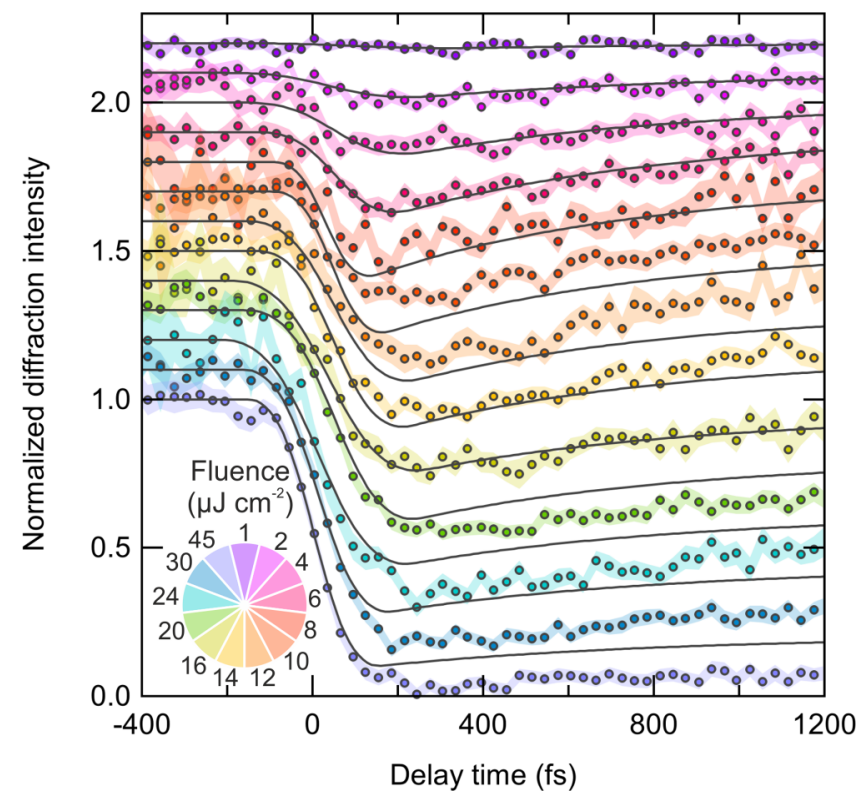

FIG. 7. Countermodel fit using literature absorption coefficient of the orbital order XRD transients. Attempt to fit the XRD transients of the orbital reflection with a fixed optical-absorption length according to literature of $z_{a}=17 \mathrm{~nm}$ [66]. While single traces may be relatively well described, an adequate description of the fluence dependence is not possible.

with the full data set. An example of such a local minimum of the fit to the data is shown in Fig. 7.

\section{APPENDIX C: MODELING OF STRUCTURAL ORDER TRANSIENTS}

We describe the dynamics of the structural order using a phenomenological fluence-dependent double-well potential model [51,73]. For a detailed model description, we refer the reader to Huber et.al. [51] - the following gives a brief outline necessary to understand the modeling and the derived parameters in this work. A summary of all model parameters can be found in Table II.

We define a ground-state double-well potential as

$$
V(x)=\frac{1}{2}\left[1-\eta_{\mathrm{exc}}(t)\right] x^{2}+\frac{1}{4} x^{4},
$$

where the excitation parameter $\eta_{\text {exc }}(t)$ relates to the laser induced suppression of the electronic potential that stabilizes

TABLE II. Overview of all model parameters used to fit the structural order transient dynamics.

\begin{tabular}{|c|c|c|c|}
\hline Variable & Unit & Description & Equation \\
\hline$n_{c, \mathrm{Str}}$ & $\mathrm{meV} / \mathrm{Ti}$ atom & critical threshold energy density of the structural order & (C6) \\
\hline$n$ & $\mathrm{meV} / \mathrm{Ti}$ atom & laser-induced energy density & (C5) \\
\hline$\eta_{\mathrm{exc}}(t)$ & & excitation parameter & (C6) \\
\hline$\tau_{\text {rel,Str }}$ & ps & relaxation-time constant of the orbital order & (C5) \\
\hline$z_{a}$ & $\mathrm{~nm}$ & optical-absorption length of the 800 -nm pump & (C3) \\
\hline$\tau_{L}$ & fs & pulse length of the optical laser ( $35 \mathrm{fs}$ ) & (C3) \\
\hline$F$ & $\mathrm{~J} / \mathrm{cm}^{2}$ & absorbed laser fluence & (C3) \\
\hline$\omega$ & $\mathrm{THz}$ & ground-state frequency of amplitude mode $(3.4 \mathrm{THz})$ & (C2) \\
\hline$\zeta$ & & phonon mode damping constant & $(\mathrm{C} 2),(\mathrm{C} 7)$ \\
\hline
\end{tabular}


the PLD. In the unexcited (ground) state, the system is separated by a Peierls barrier (at $x=0$ ) with potential minima at $x= \pm 1$, corresponding to the equilibrium PLD of the CDW phase. Upon laser induced hot-carrier injection, we assume a local change in the electronic potential landscape that effectively destabilizes the PLD by reducing (or even inverting) the quadratic contribution in Eq. (C1). The resulting structural motion to re-equilibrate the system towards the new, transient potential minimum along the related coordinate $x$ can then be determined by solving the corresponding equation of motion:

$0=\frac{1}{\omega^{2}} \frac{\partial^{2}}{\partial t^{2}} x(t)-\left[1-\eta_{\mathrm{exc}}(t)\right] x(t)+x(t)^{3}+\frac{2 \zeta(t)}{\omega} \frac{\partial}{\partial t} x(t)$.

Here, $\omega$ denotes the angular frequency of the motion at the ground-state minima of the double-well potential (for $A 1 g^{*}$ : $\omega=2 \pi f$ with $f=3.4 \mathrm{THz})[69,71]$ and $\zeta(t)$ denotes the (fluence and time) dependent damping ratio $[61,69,70]$ [note that for phonons $\zeta=\gamma(t) / f$, where $\gamma$ is the phonon damping constant (linewidth) and $f$ is the mode frequency]. For comparison to experimental data, the change in scattering intensity resulting from this formalism relates to $I(t) / I_{0} \propto|x(t)|^{2}$, where $x(t)$ is obtained by solving the differential Eq. (C2) using an ordinary differential-equation (ODE) solver.

Depending on the magnitude of the excitation, one can expect three characteristic phenomena. First, for weak excitation $\left[\eta_{\text {exc }}<1\right]$, the potential minima are slightly increased to more positive values, leading to weak amplitude structural oscillations around the ground-state equilibrium position (with angular frequency $\omega$ ). Second, for moderate excitation $\left[\eta_{\text {exc }} \approx 1\right]$, the potential barrier vanishes such that the ground-state structure is destabilized. Third, for strong excitations $\left[\eta_{\text {exc }}>1\right]$, the potential at the ground-state minima overshoots to the opposite side [leading to a $V(x)>0$ for all $x$ ] and the potential walls steepen, causing higher oscillation magnitudes and an increase in oscillation frequency (phonon hardening).

Analogous to the case of modeling the orbital order transients, we correct for (a) spatial inhomogeneity and (b) finite temporal duration of the laser excitation by taking into account (a) the optical-absorption length $z_{a}=35 \mathrm{~nm}$ and (b) the laser pulse length $\tau_{L}=35$ fs. Using the absorbed laser fluence $F$, the initial energy density $n_{0}$ at position $z_{i}$ is then written as

$$
\begin{aligned}
n_{0}\left(t, z_{i}\right) d z= & F\left(1-e^{-d z / z_{a}}\right) e^{-z_{i} / z_{a}} \\
& \times \frac{1}{2}\left[1+\operatorname{erf}\left(\frac{t \sqrt{4 \ln 2}}{\tau_{L}}\right)\right] .
\end{aligned}
$$

Based on this temporal and spatial excitation profile, we solve the differential equation of motion in Eq. (C3) for each slice along $z$ (also here, the 60-nm-thick flake is divided into $N=100$ slices of thickness $d z=0.6 \mathrm{~nm}$ ), yielding the mean displacement $x_{i}(t)$ in the ith layer. We then obtain the change in scattering intensity corrected for spatial excitation inhomogeneity for each fluence as follows:

$$
\frac{I(t)}{I_{0}}=\frac{1}{N} \sum_{i=0}^{N}\left|x_{i}(t)\right|^{2} .
$$

(a)

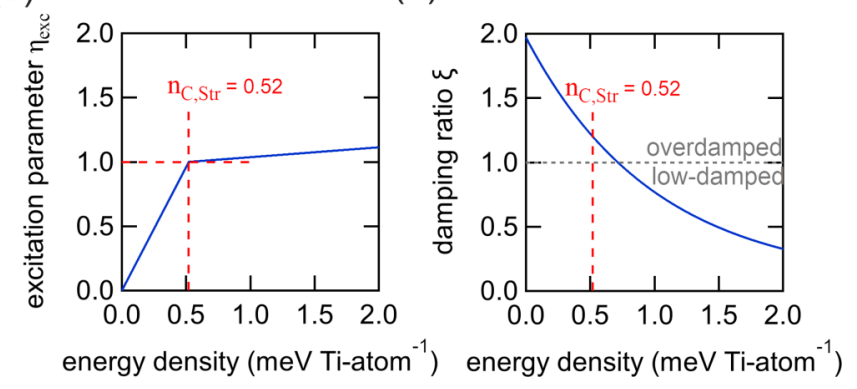

FIG. 8. Illustration of double-well potential model parameters. (a) Relation between the pump-induced energy density (horizontal axis) and the excitation parameter $\eta_{\mathrm{exc}}$, according to the formalism in Eq. (C6). (b) Relation between the pump-induced energy density (horizontal axis) and the damping constant $\zeta$, according to the formalism in Eq. (C7).

Finally, we correct for the time resolution given by the length of the x-ray probe pulse ( $15 \mathrm{fs}$, FWHM) by convolution of each time trace $\frac{I(t)}{I_{0}}$ with a corresponding Gaussian function.

In order to phenomenologically describe the experimental diffraction data, we relate two parameters to the local energy density $n(t)$ : (a) the excitation parameter $\eta_{\text {exc }}$ and (b) the damping ratio, which clearly cannot be constant as for low excitation densities the mode is overdamped, whereas the mode is clearly visible at higher densities (see Fig. 3). Therefore, we make both parameters depend on the local, time-dependent energy density $n(t)$, which relates to the initial energy density $n_{0}$ [see Eq. (C3)] via

$$
n(t)=n_{0}\left(t, z_{i}\right) e^{-t / \tau_{\mathrm{rel}, \mathrm{Str}}},
$$

where $\tau_{\text {rel,Str }}$ denotes the time constant for relaxation back to the initial double-well potential ground state. Regarding (a), we define the mathematical relation describing the excitation parameter $\eta_{\text {exc }}$ according to

$$
\eta_{\mathrm{exc}}=\left\{\begin{array}{ll}
\frac{n(t)}{n_{c, \mathrm{Str}}} & \text { for } n(t) \leqslant n_{c, \mathrm{Str}} \\
\frac{n(t)-n_{c, \mathrm{Str}}}{\alpha n_{c, \mathrm{Str}}}+1 & \text { for } n(t)>n_{c, \mathrm{Str}}
\end{array} .\right.
$$

A graphical representation of this formalism is shown in Fig. 8(a). In short, the excitation parameter $\eta_{\text {exc }}$ scales linearly until the critical energy density $n_{c, \text { Str }}$, at which the energy barrier separating the double-well potential has vanished $\left(\eta_{\mathrm{exc}}=\right.$ 1). For higher energy densities, so when the Peierls barrier stabilizing the PLD is suppressed, the excitation parameter $\eta_{\text {exc }}$ saturates: it still scales linearly, but with shallower slope [see variable $\alpha$ in Eq. (C6)].

Regarding (b), the damping ratio $\zeta(t)$ is described by an exponentially decaying fluence according to

$$
\zeta(t)=\zeta_{0}+\zeta_{1} e^{-n(t)} .
$$

A graphical representation of this formalism is shown in Fig. 8(b). In short, this damping term contains two fluence regimes: (i) a low-fluence overdamped regime $\left(\zeta=\zeta_{0}+\right.$ $\left.\zeta_{1}>1\right)$ and (ii) a high-fluence low-damped regime $\left(\zeta=\zeta_{0}<\right.$ 1 ), where $\zeta$ denotes the damping ratio. It should be noted that this mathematical formalism was chosen as simple as possible yet still yielding a best possible phenomenological description of the experimental data. 


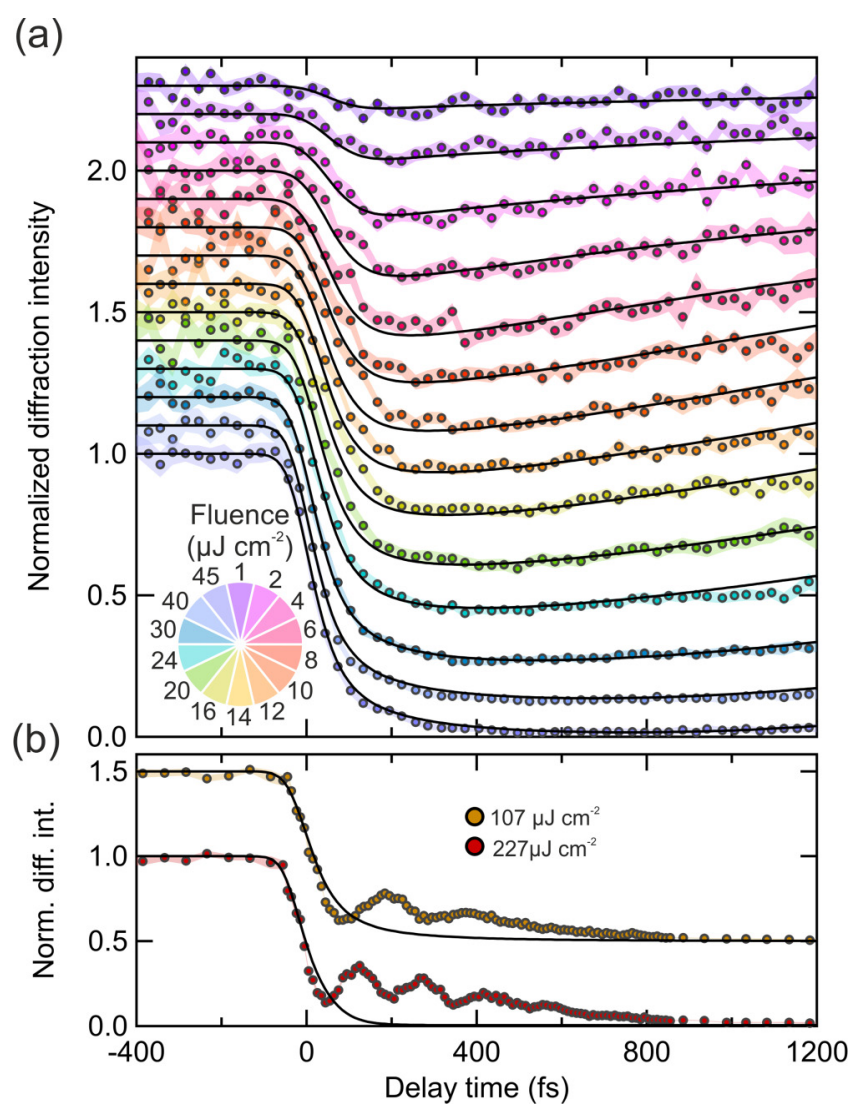

FIG. 9. Simplified double-potential model fit of the structural order XRD transients. Normalized XRD transients of the structural $(5 / 21 / 23 / 2)$ reflection at $12.620 \mathrm{keV}$ (see colored markers for increasing fluence-curves are offset for fluences below $45 \mu \mathrm{J} / \mathrm{cm}^{2}$ for better visibility) together with the fits (black curves) of the simplified double-well-potential model. This simplified model uses the same values for $n_{c, \text { Str }}, \alpha$ and $\tau_{\text {rel,Str }}$ as in Fig. 3, but a constant damping ratio of $\zeta(t)=1$. While this simpler model reproduces well the low-fluence regime associated with melting of the PLD (a), it does not reproduce the coherent oscillations in the high-fluence regime (b).

Given the above relations, we manually optimize model parameters by comparison of model data with all traces as function of laser fluence simultaneously. The resulting time traces together with experimental data are shown in Figs. 3(a) and 3(b). We find best agreement of model and experimental data using a ground-state destabilization energy $n_{c, \mathrm{Str}}=$ $0.52 \mathrm{meV}$ per Ti atom as well as $\alpha=25$ [see Eq. (C6)], $\tau_{\text {rel,Str }}=1.6 \mathrm{ps}, \zeta_{0}=0.07$, and $\zeta_{1}=1.9$ [see Eq. (C7)]. Note that the obtained value ground-state destabilization energy $n_{c \text {,Str }}$ refers to the scenario at which the potential wells have vanished and the PLD is destabilized. For comparison to the literature time-resolved ARPES data [28,34,61] (which probes only the first atomic monolayers), the critical energy is reached in the top-most surface layer at excitation fluences of $4.8 \mu \mathrm{J} / \mathrm{cm}^{2}(0.52 \mathrm{meV}$ per Ti atom).

We emphasize that the presented model was chosen for the best possible description of the experimental data while keeping the model formalisms simple. In this regard, we have to discuss the influence of the phenomenological cho-

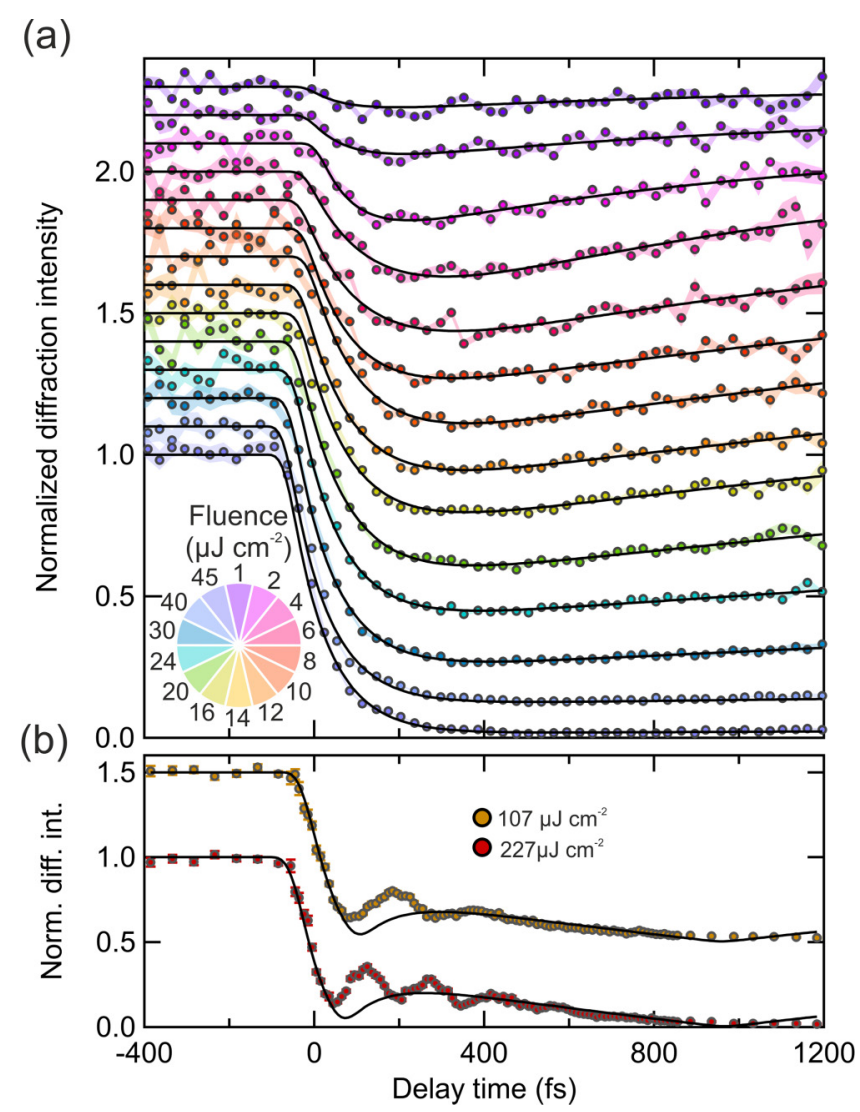

FIG. 10. Double-exponential model fit of the structural order XRD transients. Normalized XRD transients of the structural $(5 / 2$ $1 / 23 / 2$ ) reflection at $12.620 \mathrm{keV}$ (see colored markers for increasing fluence-curves are offset for fluences below $45 \mu \mathrm{J} / \mathrm{cm}^{2}$ for better visibility) together with the fits (black curves) of the doubleexponential model according to literature [28,34]. The decay values extracted from the fits that describe the relative signal decrease (corresponding to the melting dynamics of the orbital and structural order) are shown in Fig. 4(b).

sen damping ratio $\zeta(t)$ [see Eq. (C7)]. To confirm that the critical energy density $n_{c, \text { Str }}$ associated with suppression of the double-well potential is quantitatively not affected by the choice of this damping term, we simulate the same model [using the same values for $n_{\mathrm{c}, \mathrm{Str}}, \alpha$ and $\tau_{\text {rel,Str }}$ as above] but assuming a constant damping ratio of $\zeta(t)=1$. As seen in Fig. 9, the model still describes well the low-fluence $(1<$ $\left.F<20 \mu \mathrm{J} / \mathrm{cm}^{2}\right)$ tr-XRD intensities, in which the transition from the PLD stabilizing double-well potential to a parabolic destabilized state occurs. However, it is apparent that this simple model does not reproduce the oscillatory component of the high-fluence $\left(F>30 \mu \mathrm{J} / \mathrm{cm}^{2}\right)$ tr-XRD intensities [Fig. 9(b)]. In contrast, our more sophisticated formalism [see Eq. (C7)] yields good agreement of the complete data set, including the observed hardening of the $A 1 g^{*}$ mode with increasing fluence (see Fig. 3), yet the quantitative result related to the critical melting threshold of the PLD $n_{c, \text { Str }}$ remains consistent (see Fig. 10). 
[1] K. W. Kim, A. Pashkin, H. Schäfer, M. Beyer, M. Porer, T. Wolf, C. Bernhard, J. Demsar, R. Huber, and A. Leitenstorfer, Ultrafast transient generation of spin-density-wave order in the normal state of $\mathrm{BaFe}_{2} \mathrm{As}_{2}$ driven by coherent lattice vibrations, Nat. Mater. 11, 497 (2012).

[2] C. Dornes, Y. Acremann, M. Savoini, M. Kubli, M. J. Neugebauer, E. Abreu, L. Huber, G. Lantz, C. A. F. Vaz, H. Lemke et al., The ultrafast einstein-de haas effect, Nature (London) 565, 209 (2019).

[3] R. V. Mikhaylovskiy, E. Hendry, A. Secchi, J. H. Mentink, M. Eckstein, A. Wu, R. V. Pisarev, V. V. Kruglyak, M. I. Katsnelson, T. Rasing et al., Ultrafast optical modification of exchange interactions in iron oxides, Nat. Commun. 6, 8190 (2015).

[4] D. Fausti, R. I. Tobey, N. Dean, S. Kaiser, A. Dienst, M. C. Hoffmann, S. Pyon, T. Takayama, H. Takagi, and A. Cavalleri, Light-induced superconductivity in a stripe-ordered cuprate, Science 331, 189 (2011).

[5] M. Rini, R. Tobey, N. Dean, J. Itatani, Y. Tomioka, Y. Tokura, R. W. Schoenlein, and A. Cavalleri, Control of the electronic phase of a manganite by mode-selective vibrational excitation, Nature (London) 449, 72 (2007).

[6] B. Sipos, A. F. Kusmartseva, A. Akrap, H. Berger, L. Forró, and E. Tutiš, From mott state to superconductivity in $1 \mathrm{t}-\mathrm{TaS}_{2}$, Nat. Mater. 7, 960 (2008).

[7] H. Seiler, S. Palato, C. Sonnichsen, H. Baker, E. Socie, D. P. Strandell, and P. Kambhampati, Two-dimensional electronic spectroscopy reveals liquid-like lineshape dynamics in $\mathrm{CsPbI}_{2}$ perovskite nanocrystals, Nat. Commun. 10, 4962 (2019).

[8] K. Miyata, D. Meggiolaro, M. Tuan Trinh, P. P. Joshi, E. Mosconi, S. C. Jones, F. De Angelis, and X. Y. Zhu, Large polarons in lead halide perovskites, Sci. Adv. 3, e1701217 (2017).

[9] C. Lian, S. J. Zhang, S. Q. Hu, M. X. Guan, and S. Meng, Ultrafast charge ordering by self-amplified exciton-phonon dynamics in $\mathrm{TiSe}_{2}$, Nat. Commun. 11, 43 (2020).

[10] A. Lanzara, P. V. Bogdanov, X. J. Zhou, S. A. Kellar, D. L. Feng, E. D. Lu, T. Yoshida, H. Eisaki, A. Fujimori, K. Kishio et al., Evidence for ubiquitous strong electron-phonon coupling in high-temperature superconductors, Nature (London) 412, 510 (2001).

[11] E. Pastor, J. S. Park, L. Steier, S. Kim, M. Grätzel, J. R. Durrant, A. Walsh, and A. A. Bakulin, In situ observation of picosecond polaron self-localisation in $\alpha-\mathrm{Fe}_{2} \mathrm{O}_{3}$ photoelectrochemical cells, Nat. Commun. 10, 3962 (2019).

[12] C. Boeglin, E. Beaurepaire, V. Halté, V. López-Flores, C. Stamm, N. Pontius, H. A. Dürr, and J. Y. Bigot, Distinguishing the ultrafast dynamics of spin and orbital moments in solids, Nature (London) 465, 458 (2010).

[13] E. Morosan, H. W. Zandbergen, B. S. Dennis, J. W. G. Bos, Y. Onose, T. Klimczuk, A. P. Ramirez, N. P. Ong, and R. J. Cava, Superconductivity in $\mathrm{Cu}_{\mathrm{X}} \mathrm{TiSe}_{2}$, Nat. Phys. 2, 544 (2006).

[14] A. F. Kusmartseva, B. Sipos, H. Berger, L. Forró, and E. Tutiš, Pressure Induced Superconductivity In Pristine 1T-TiSe ${ }_{2}$, Phys. Rev. Lett. 103, 236401 (2009).

[15] K. C. Woo, F. C. Brown, W. L. McMillan, R. J. Miller, M. J. Schaffman, and M. P. Sears, Superlattice formation in titanium diselenide, Phys. Rev. B 14, 3242 (1976).

[16] J. A. Holy, K. C. Woo, M. V. Klein, and F. C. Brown, Raman and infrared studies of superlat- tice formation in $\mathrm{TiSe}_{2}$, Phys. Rev. B 16, 3628 (1977).

[17] F. J. Di Salvo, D. E. Moncton, and J. V. Waszczak, Electronic properties and superlattice formation in the semimetal $\mathrm{TiSe}_{2}$, Phys. Rev. B 14, 4321 (1976).

[18] D. Qian, D. Hsieh, L. Wray, E. Morosan, N. L. Wang, Y. Xia, R. J. Cava, and M. Z. Hasan, Emergence of Fermi Pockets in a New Excitonic Charge-Density-Wave Melted Superconductor, Phys. Rev. Lett. 98, 117007 (2007).

[19] M. D. Watson, O. J. Clark, F. Mazzola, I. Marković, V. Sunko, T. K. Kim, K. Rossnagel, and P. D. C. King, Orbital- and Kz -Selective Hybridization of Se $4 p$ and Ti $3 d$ States in the Charge Density Wave Phase of $\mathrm{TiSe}_{2}$, Phys. Rev. Lett. 122, 076404 (2019).

[20] H. Cercellier, C. Monney, F. Clerc, C. Battaglia, L. Despont, M. G. Garnier, H. Beck, P. Aebi, L. Patthey, H. Berger et al., Evidence for an Excitonic Insulator Phase in 1T-TiSe ${ }_{2}$, Phys. Rev. Lett. 99, 146403 (2007).

[21] T. Pillo, J. Hayoz, H. Berger, and F. Lévy, Photoemission of bands above the fermi level: The excitonic insulator phase transition, Phys. Rev. B 61, 16213 (2000).

[22] M. D. Watson, A. M. Beales, and P. D. C. King, On the origin of the anomalous peak in the resistivity of $\mathrm{TiSe}_{2}$, Phys. Rev. B 99, 195142 (2019).

[23] G. Li, W. Z. Hu, D. Qian, D. Hsieh, M. Z. Hasan, E. Morosan, R. J. Cava, and N. L. Wang, Semimetal-to-Semimetal Charge Density Wave Transition in 1T-TiSe ${ }_{2}$, Phys. Rev. Lett. 99, 027404 (2007).

[24] M. L. Mottas, T. Jaouen, B. Hildebrand, M. Rumo, F. Vanini, E. Razzoli, E. Giannini, C. Barreteau, D. R. Bowler, C. Monney et al., Semimetal-to-semiconductor transition and chargedensity-wave suppression in $1 \mathrm{~T}-\mathrm{TiSe}_{2-\mathrm{X}} \mathrm{S}_{\mathrm{X}}$ single crystals, Phys. Rev. B 99, 155103 (2019).

[25] H. P. Hughes, Structural distortion in $\mathrm{TiSe}_{2}$ and related materials-a possible jahn-teller effect? J. Phys. C: Solid State Phys. 10, L319 (1977).

[26] W. Y. Liang, in Intercalation Layer Matter (Springer, Boston, 1986), pp. 31-73.

[27] C. Monney, C. Battaglia, H. Cercellier, P. Aebi, and H. Beck, Exciton Condensation Driving the Periodic Lattice Distortion of 1T-TiSe ${ }_{2}$, Phys. Rev. Lett. 106, 106404 (2011).

[28] T. Rohwer, S. Hellmann, M. Wiesenmayer, C. Sohrt, A. Stange, B. Slomski, A. Carr, Y. Liu, L. M. Avila, M. Kalläsignne et al., Collapse of long-range charge order tracked by time-resolved photoemission at high momenta, Nature (London) 471, 490 (2011).

[29] T. Jaouen, M. Rumo, B. Hildebrand, M.-L. Mottas, C. W. Nicholson, G. Kremer, B. Salzmann, F. Vanini, C. Barreteau, E. Giannini et al., Unveiling the semimetallic nature of $1 \mathrm{~T}-\mathrm{TiSe}_{2}$ by doping its charge density wave, arXiv:1911.06053.

[30] C. Monney, G. Monney, P. Aebi, and H. Beck, Electron-hole instability in 1T-TiSe 2 , New J. Phys. 14, 075026 (2012).

[31] M. Hellgren, J. Baima, R. Bianco, M. Calandra, F. Mauri, and L. Wirtz, Critical Role of the Exchange Interaction for the Electronic Structure and Charge-Density-Wave Formation in $\mathrm{TiSe}_{2}$, Phys. Rev. Lett. 119, 176401 (2017).

[32] C. Monney, H. Cercellier, F. Clerc, C. Battaglia, E. F. Schwier, C. Didiot, M. G. Garnier, H. Beck, P. Aebi, H. Berger et al., Spontaneous exciton condensation in $1 \mathrm{~T}-\mathrm{TiSe}_{2}$ : BCS-like approach, Phys. Rev. B 79, 045116 (2009). 
[33] G. Monney, C. Monney, B. Hildebrand, P. Aebi, and H. Beck, Impact of Electron-Hole Correlations on the 1T-TiSe ${ }_{2}$ Electronic Structure, Phys. Rev. Lett. 114, 086402 (2015).

[34] S. Mathias, S. Eich, J. Urbancic, S. Michael, A. V. Carr, S. Emmerich, A. Stange, T. Popmintchev, T. Rohwer, M. Wiesenmayer et al., Self-amplified photo-induced gap quenching in a correlated electron material, Nat. Commun. 7, 12902 (2016).

[35] A. Kogar, M. S. Rak, S. Vig, A. A. Husain, F. Flicker, Y. Il Joe, L. Venema, G. J. MacDougall, T. C. Chiang, E. Fradkin et al., Signatures of exciton condensation in a transition metal dichalcogenide, Science 358, 1314 (2017).

[36] J.-P. Castellan, S. Rosenkranz, R. Osborn, Q. Li, K. E. Gray, X. Luo, U. Welp, G. Karapetrov, J. P. C. Ruff, and J. van Wezel, Chiral Phase Transition in Charge Ordered 1T-TiSe 2 , Phys. Rev. Lett. 110, 196404 (2013).

[37] S. Y. Xu, Q. Ma, Y. Gao, A. Kogar, A. Zong, A. M. Mier Valdivia, T. H. Dinh, S. M. Huang, B. Singh, C. H. Hsu et al., Spontaneous gyrotropic electronic order in a transition-metal dichalcogenide, Nature (London) 578, 545 (2020).

[38] B. Hildebrand, T. Jaouen, M. L. Mottas, G. Monney, C. Barreteau, E. Giannini, D. R. Bowler, and P. Aebi, Local RealSpace View of the Achiral 1T-TiSe $2 \times 2 \times 2$ Charge Density Wave, Phys. Rev. Lett. 120, 136404 (2018).

[39] S. Rosenkranz, R. Osborn, and J. van Wezel, Rosenkranz, Osborn, and van Wezel Reply, Phys. Rev. Lett. 122, 229702 (2019).

[40] M. K. Lin, J. A. Hlevyack, P. Chen, R. Y. Liu, and T. C. Chiang, Comment on " $\mathrm{C}$ Hiral Phase Transition in Charge Ordered 1T-TiSe 2 ," Phys. Rev. Lett. 122, 229701 (2019).

[41] H. Ueda, M. Porer, J. R. L. Mardegan, S. Parchenko, N. Grurung, F. Fabrizi, M. Ramakrishnan, L. Boie, M. J. Neugebauer, B. Burganov et al., Correlation between electronic and structural orders in 1T-TiSe ${ }_{2}$, arXiv:2006.08983.

[42] T. Kaneko, Y. Ohta, and S. Yunoki, Exciton-phonon cooperative mechanism of the triple- q charge-density-wave and antiferroelectric electron polarization in $\mathrm{TiSe}_{2}$, Phys. Rev. B 97, 155131 (2018).

[43] J. van Wezel, P. Nahai-Williamson, and S. S. Saxena, Excitonphonon-driven charge density wave in $\mathrm{TiSe}_{2}$, Phys. Rev. B 81, 165109 (2010).

[44] J. van Wezel, P. Nahai-Williamson, and S. S. Saxena, An alternative interpretation of recent arpes measurements on $\mathrm{TiSe}_{2}$, Europhys. Lett. 89, 47004 (2010).

[45] C. Chen, B. Singh, H. Lin, and V. M. Pereira, Reproduction of the Charge Density Wave Phase Diagram in 1T-TiSe 2 Exposes its Excitonic Character, Phys. Rev. Lett. 121, 226602 (2018).

[46] V. Scagnoli, U. Staub, A. M. Mulders, M. Janousch, G. I. Meijer, G. Hammerl, J. M. Tonnerre, and N. Stojic, Role of magnetic and orbital ordering at the metal-insulator transition in $\mathrm{NdNiO}_{3}$, Phys. Rev. B 73, 100409 (2006).

[47] U. Staub, G. I. Meijer, F. Fauth, R. Allenspach, J. G. Bednorz, J. Karpinski, S. M. Kazakov, L. Paolasini, and F. D’Acapito, Direct observation of charge order in an epitaxial $\mathrm{NdNiO}_{3}$ film, Phys. Rev. Lett. 88, 126402 (2002).

[48] G. Ghiringhelli, M. Le Tacon, M. Minola, S. Blanco-Canosa, C. Mazzoli, N. B. Brookes, G. M. De Luca, A. Frano, D. G. Hawthorn, F. He et al., Long-range incommensurate charge fluctuations in $(\mathrm{Y}, \mathrm{Nd}) \mathrm{Ba}_{2} \mathrm{Cu}_{3} \mathrm{O}_{6+\mathrm{x}}$, Science 337, 821 (2012).
[49] V. Scagnoli, U. Staub, Y. Bodenthin, R. A. De Souza, M. García-Fernández, M. Garganourakis, A. T. Boothroyd, D. Prabhakaran, and S. W. Lovesey, Observation of orbital currents in $\mathrm{CuO}$, Science 332, 696 (2011).

[50] Y. Murakami, J. P. Hill, D. Gibbs, M. Blume, I. Koyama, M. Tanaka, H. Kawata, T. Arima, Y. Tokura, K. Hirota et al., Resonant X-Ray Scattering from Orbital Ordering in $\mathrm{LaMnO}_{3}$, Phys. Rev. Lett. 81, 582 (1998).

[51] T. Huber, S. O. Mariager, A. Ferrer, H. Schäfer, J. A. Johnson, S. Grübel, A. Lübcke, L. Huber, T. Kubacka, C. Dornes et al., Coherent Structural Dynamics of a Prototypical Charge-Density-Wave-to-Metal Transition, Phys. Rev. Lett. 113, 026401 (2014).

[52] V. Esposito, L. Rettig, E. M. Bothschafter, Y. Deng, C. Dornes, L. Huber, T. Huber, G. Ingold, Y. Inubushi, T. Katayama et al., Dynamics of the photoinduced insulator-to-metal transition in a nickelate film, Struct. Dyn. 5, 064501 (2018).

[53] S. de Jong, R. Kukreja, C. Trabant, N. Pontius, C. F. Chang, T. Kachel, M. Beye, F. Sorgenfrei, C. H. Back, B. Bräuer et al., Speed limit of the insulator-metal transition in magnetite, Nat. Mater. 12, 882 (2013).

[54] E. Möhr-Vorobeva, S. L. Johnson, P. Beaud, U. Staub, R. De Souza, C. Milne, G. Ingold, J. Demsar, H. Schaefer, and A. Titov, Nonthermal Melting of a Charge Density Wave in $\mathrm{TiSe}_{2}$, Phys. Rev. Lett. 107, 036403 (2011).

[55] M. Porer, M. Fechner, M. Kubli, M. J. Neugebauer, S. Parchenko, V. Esposito, A. Narayan, N. A. Spaldin, R. Huber, M. Radovic et al., Ultrafast transient increase of oxygen octahedral rotations in a perovskite, Phys. Rev. Res. 1, 012005 (2019).

[56] P. Beaud, S. L. Johnson, E. Vorobeva, U. Staub, R. A. De Souza, C. J. Milne, Q. X. Jia, and G. Ingold, Ultrafast structural phase transition driven by photoinduced melting of charge and orbital order, Phys. Rev. Lett. 103, 155702 (2009).

[57] T. Ishikawa, H. Aoyagi, T. Asaka, Y. Asano, N. Azumi, T. Bizen, H. Ego, K. Fukami, T. Fukui, Y. Furukawa et al., A compact X-ray free-electron laser emitting in the sub-ångström region, Nat. Photonics 6, 540 (2012).

[58] T. Katayama, S. Owada, T. Togashi, K. Ogawa, P. Karvinen, I. Vartiainen, A. Eronen, C. David, T. Sato, K. Nakajima et al., A beam branching method for timing and spectral characterization of hard x-ray free-electron lasers, Struct. Dyn. 3, 034301 (2016).

[59] K. Nakajima, Y. Joti, T. Katayama, S. Owada, T. Togashi, T. Abe, T. Kameshima, K. Okada, T. Sugimoto, M. Yamaga et al., Software for the data analysis of the arrivaltiming monitor at SACLA, J. Synchrotron Radiat. 25, 592 (2018).

[60] M. Porer, U. Leierseder, J.-M. Ménard, H. Dachraoui, L. Mouchliadis, I. E. Perakis, U. Heinzmann, J. Demsar, K. Rossnagel, and R. Huber, Non-thermal separation of electronic and structural orders in a persisting charge density wave, Nat. Mater. 13, 857 (2014).

[61] H. Hedayat, C. J. Sayers, D. Bugini, C. Dallera, D. Wolverson, T. Batten, S. Karbassi, S. Friedemann, G. Cerullo, J. van Wezel et al., Excitonic and lattice contributions to the charge density wave in $1 \mathrm{~T}-\mathrm{TiSe}_{2}$ revealed by a phonon bottleneck, Phys. Rev. Res. 1, 023029 (2019).

[62] S. Kitou, A. Nakano, S. Kobayashi, K. Sugawara, N. Katayama, N. Maejima, A. Machida, T. Watanuki, K. Ichimura, S. 
Tanda et al., Effect of $\mathrm{Cu}$ intercalation and pressure on excitonic interaction in 1T-TiSe 2 , Phys. Rev. B 99, 104109 (2019).

[63] Note that the observed dynamics are approximately three times slower than the experimental time resolution $(\sim 50 \mathrm{fs}$, FWHM; see Experimental section), ruling out immediate effects of hotcarrier injection on the Se $4 p$ orbital geometry and the hole population.

[64] P. Beaud, A. Caviezel, S. O. Mariager, L. Rettig, G. Ingold, C. Dornes, S. W. Huang, J. A. Johnson, M. Radovic, T. Huber et al., A time-dependent order parameter for ultrafast photoinduced phase transitions, Nat. Mater. 13, 923 (2014).

[65] M. Porer, L. Rettig, E. M. Bothschafter, V. Esposito, R. B. Versteeg, P. H. M. van Loosdrecht, M. Savoini, J. Rittmann, M. Kubli, G. Lantz et al., Correlations between electronic order and structural distortions and their ultrafast dynamics in the singlelayer manganite $\operatorname{Pr}_{0.5} \mathrm{Ca}_{1.5} \mathrm{MnO}_{4}$, Phys. Rev. B 101, 075119 (2020).

[66] S. C. Bayliss and W. Y. Liang, Reflectivity, Joint density of states and band structure of group ivb transition-metal dichalcogenides, J. Phys. C: Solid State Phys. 18, 3327 (1985).

[67] Note that the absorbed fluence of $33 \mu \mathrm{J} / \mathrm{cm}^{2}$ corresponds to excitation densities of only $2.4 \times 10^{-3}$ photons per Ti atom, rendering the contribution of hole depopulation on the scattering intensity negligible.

[68] N. Wakabayashi, H. G. Smith, K. C. Woo, and F. C. Brown, Phonons and charge density waves in $1 \mathrm{~T}-\mathrm{TiSe}_{2}$, Solid State Commun. 28, 923 (1978).

[69] M. Holt, P. Zschack, H. Hong, M. Y. Chou, and T. C. Chiang, $\mathrm{X}$-Ray Studies of Phonon Softening in TiSe ${ }_{2}$, Phys. Rev. Lett. 86, 3799 (2001).

[70] F. Weber, S. Rosenkranz, J. P. Castellan, R. Osborn, G. Karapetrov, R. Hott, R. Heid, K. P. Bohnen, and A. Alatas, Electron-Phonon Coupling and the Soft Phonon Mode in $\mathrm{TiSe}_{2}$, Phys. Rev. Lett. 107, 266401 (2011).

[71] C. S. Snow, J. F. Karpus, S. L. Cooper, T. E. Kidd, and T. C. Chiang, Quantum Melting of the Charge-Density-Wave State in 1T-TiSe 2 , Phys. Rev. Lett. 91, 136402 (2003).

[72] S. Hellmann, T. Rohwer, M. Kalläne, K. Hanff, C. Sohrt, A. Stange, A. Carr, M. M. Murnane, H. C. Kapteyn, L. Kipp et al., Time-domain classification of charge-density-wave insulators, Nat. Commun. 3, 1069 (2012).

[73] R. Yusupov, T. Mertelj, V. V. Kabanov, S. Brazovskii, P. Kusar, J. H. Chu, I. R. Fisher, and D. Mihailovic, Coherent dynamics of macroscopic electronic order through a symmetry breaking transition, Nat. Phys. 6, 681 (2010).
[74] Also in this case, we cannot find agreement of model and all experimental traces when using the literature absorption coefficient.

[75] R. Bianco, M. Calandra, and F. Mauri, Electronic and vibrational properties of $\mathrm{TiSe}_{2}$ in the charge-density-wave phase from first principles, Phys. Rev. B 92, 094107 (2015).

[76] P. Chen, Y.-H. Chan, X.-Y. Fang, S.-K. Mo, Z. Hussain, A.-V. Fedorov, M. Y. Chou, and T.-C. Chiang, Hidden order and dimensional crossover of the charge density waves in $\mathrm{TiSe}_{2}$, Sci. Rep. 6, 37910 (2016).

[77] P. Knowles, B. Yang, T. Muramatsu, O. Moulding, J. Buhot, C. J. Sayers, E. Da Como, and S. Friedemann, Fermi Surface Reconstruction and Electron Dynamics at the Charge-DensityWave Transition in $\mathrm{TiSe}_{2}$, Phys. Rev. Lett. 124, 167602 (2020).

[78] R. Huber, F. Tauser, A. Brodschelm, M. Bichler, G. Abstreiter, and A. Leitenstorfer, How many-particle interactions develop after ultrafast excitation of an electron-hole plasma, Nature (London) 414, 286 (2001).

[79] D. H. Torchinsky, F. Mahmood, A. T. Bollinger, I. Božović, and N. Gedik, Fluctuating charge-density waves in a cuprate superconductor, Nat. Mater. 12, 387 (2013).

[80] S. Manzeli, D. Ovchinnikov, D. Pasquier, O. V. Yazyev, and A. Kis, 2D transition metal dichalcogenides, Nat. Rev. Mater. 2, 17033 (2017)

[81] M. Hepting, R. J. Green, Z. Zhong, M. Bluschke, Y. E. Suyolcu, S. Macke, A. Frano, S. Catalano, M. Gibert, R. Sutarto et al., Complex magnetic order in nickelate slabs, Nat. Phys. 14, 1097 (2018).

[82] R. Ramesh and D. G. Schlom, Creating emergent phenomena in oxide superlattices, Nat. Rev. Mater. 4, 257 (2019).

[83] G. Rainò, M. A. Becker, M. I. Bodnarchuk, R. F. Mahrt, M. V. Kovalenko, and T. Stöferle, Superfluorescence from lead halide perovskite quantum dot superlattices, Nature (London) 563, 671 (2018).

[84] Z. Luo, T. P. Dao, A. Hrabec, J. Vijayakumar, A. Kleibert, M. Baumgartner, E. Kirk, J. Cui, T. Savchenko, G. Krishnaswamy et al., Chirally coupled nanomagnets, Science 363, 1435 (2019).

[85] S. H. Skjærvø, C. H. Marrows, R. L. Stamps, and L. J. Heyderman, Advances in artificial spin ice, Nat. Rev. Phys. 2, 13 (2020).

[86] M. Burian, M. Porer, J. R. L. Mardegan, V. Esposito, S. Parchenko, B. Bulat, N. Gurung, M. Ramakrishnan, V. Scagnoli, H. Ueda et al., Structural involvement in the melting of the charge density wave in $1 \mathrm{~T}-\mathrm{TiSe}_{2}$, Mater. Cloud Arch. 2021.26 (2021).

Correction: The omission of a support statement in the Acknowledgment section has been fixed. 